\title{
Dinámica económica y migración en el centro de México: impronta territorial de dos procesos convergentes
}

\section{Economic dynamic and internal migration in central Mexico: territorial mark of two convergent processes}

\author{
Guillermo Olivera-Lozano \\ Carlos Galindo-PÉrez*
}

\begin{abstract}
This paper addresses the economic base of the municipalities and states of central Mexico, and their influence on the migration flows inside them. The first part is a summary of recent works in Latin America and Mexico of the migration and development relation. Secondly, the economic structure and dynamics of municipalities and metropolitan areas is examined by main economic sector. In the third section, the interregional migration flows and some characteristics of migrants are shown to illustrate the occupational aspect of migration that other studies have.
\end{abstract}

Keywords: Central Mexico, internal migration, metropolitan economy.

\begin{abstract}
Resumen
Esta investigación aborda la base económica de municipios y estados del centro del país, y su influencia sobre los flujos migratorios internos. Primero se hace una síntesis de estudios recientes en Latinoamérica y México sobre migración y desarrollo; después se analiza la estructura y dinámica económica por municipio y aglomeraciones metropolitanas, por gran sector económico; posteriormente, se muestran los destinos interregionales de la migración y algunas características de los migrantes, para ilustrar la faceta predominantemente laboral de la migración que otros análisis hacen evidente, y que aquí se reafirma, sin desconocer otros factores. Finalmente se especifican las conclusiones a las que se llegaron con este análisis.
\end{abstract}

Palabras clave: México central, migración interna, economía metropolitana.

*Universidad Nacional Autónoma de México, México. Correo-e: gol@servidor.unam.mx, carlosgp@correo.crim.unam.mx 


\section{Introducción $^{1}$}

Esta investigación aporta elementos empíricos para un mejor conocimiento de la base económica de los municipios y entidades que integran la región Centro del país -principal espacio económico, demográfico y metropolitano a nivel nacional-, para reconocer la influencia que esto tiene en la generación de flujos migratorios interestatales e intermunicipales. En la primera sección se elabora una síntesis de las investigaciones más recientes y relevantes sobre la relación migración-desarrollo en Latinoamérica y México; en la segunda parte se analiza la estructura y dinámica económica de la región a escala municipal y en sus principales aglomeraciones metropolitanas, por grandes sectores económicos; en el tercer apartado se muestran los principales destinos interregionales de la migración, principalmente metropolitanos, y se revisan algunas características de los migrantes, en particular género y estructura por edad, para ilustrar la faceta laboral de la migración que ya otras investigaciones hacen evidente, pero que la información disponible hasta hace poco no permitía calificar con precisión. Por último, se concluye con una síntesis de los principales hallazgos.

Si bien, el enfoque del estudio se centra en la dinámica económica como un factor explicativo de la migración interna, no se omiten los factores de carácter demográfico, y sobre todo urbano, que motivan los desplazamientos de la población, particularmente en las ciudades más importantes a nivel nacional que, por su carácter metropolitano, presentan una fuerte movilidad poblacional. Así, la hipótesis de este análisis es que la migración interna en la región Centro del país tiene causas económicolaborales y urbano-demográficas, así como una dimensión metropolitana, influida por un proceso de desconcentración-concentrada a escala regional que tienen la actividad económica y la población. Su intensidad, por otra parte, es diferenciada con base en el dinamismo económico de cada ciudad y los atributos de edad, nivel educativo, nivel de ingresos y género de las personas. En general, los flujos de población se dirigen hacia los espacios de mayor dinamismo económico y el sector terciario es el sector motriz de la economía de la región Centro.

${ }^{1}$ Los autores agradecen las observaciones de los dictaminadores anónimos. 


\section{Migración y desarrollo: argumentación teórica y validación empírica}

\subsection{Los avances recientes en la perspectiva latinoamericana}

En los estudios sobre migración interna, tradicionalmente se ha considerado su vinculación con el desarrollo, de acuerdo con ello, las principales causas de los movimientos de población son las desigualdades socioeconómicas, y por ende, la búsqueda de mejores condiciones de vida por parte de los migrantes. Este esquema tuvo un poder explicativo absoluto hasta los años ochenta del siglo xx, mientras la migración campo-ciudad fue el tipo de flujo poblacional predominante; sin embargo, a raíz de la reestructuración de la economía mundial y de sus regiones $-\mathrm{a}$ fines de ese mismo siglo-, y del predominio de la población urbana sobre la rural junto con un proceso de expansión metropolitana de las grandes urbes, así como del efecto de la transición demográfica en curso, el abanico de causas específicas de la migración se diversificó, haciendo más complejo su estudio.

Desde el punto de vista del desarrollo, las condiciones de dinamismo y rezago económico bastaban para explicar los flujos de población desde regiones menos desarrolladas a más civilizadas; no obstante, también han sido permanentes las críticas sobre el particular. Aroca, (2004) y Rodríguez (2004, en CEPAL, 2007) por ejemplo, refieren que ese enfoque privilegió las fuerzas de expulsión en el lugar de origen, y a la búsqueda de mayores ingresos como causa fundamental. Ellos mismos reconocen que en la actualidad existen otros motivos como el cambio de residencia y la búsqueda de mejoras en el hábitat, o la reducción de tiempos de traslado de la vivienda al trabajo, lo cual se favorece por el tipo de urbanización extendida actualmente dominante. Adicionalmente señalan que la relación migración-desarrollo no aplica en casos en los que el progreso de algunas regiones receptoras es reciente, o en regiones metropolitanas en proceso de suburbanización o desconcentración que, de cualquier manera, expulsan gente por falta de espacio, por deterioro de la calidad de vida, o por efecto de la regulación y las políticas urbanas.

Con base en lo señalado, se distingue la persistencia de factores causantes de la migración interna de tipo tradicional, asociados al desarrollo en su sentido más general, junto con factores relativamente emergentes, vinculados a la urbanización en su etapa metropolitana y regional, y a las deseconomías que de ello se han derivado. No puede considerarse que los segundos sustituyan a los primeros sino que coexisten, de lo cual deriva la complejidad de la migración que es, al mismo tiempo, un fenómeno sociodemográfico y económico en permanente transformación. 
Entre los aspectos de la relación migración-desarrollo que siguen prevaleciendo se mantiene la búsqueda de mejores condiciones de vida, en donde destacan en específico las condiciones laborales y las remuneraciones como principales detonantes, lo que a su vez se vincula con ciertas características de la población en términos de género, edad y nivel educativo. La principal conclusión en este sentido es que la migración es selectiva y que estaría favoreciendo una convergencia en el crecimiento demográfico o distribución espacial de la población (Rodríguez, 2004), al mismo tiempo que produce cambios en su perfil (CEPAL, 2007; Arroyo, 2001).

Si bien, con los datos de los censos demográficos no es posible saber si la población que migra mejora sus condiciones de vida, sí están documentadas, en cambio, las relaciones entre migración y características de la población a nivel de América Latina. Se sabe que la población de estratos jóvenes en edad laboral es la más propensa a migrar, aun cuando hay evidencias de cierta importancia de la migración de retorno (CEPAL, 2007). También se sabe que migran más las mujeres ${ }^{2}$ y la población con niveles educativos superiores al promedio, así como se ha identificado la relación que existe entre la migración y el ciclo de vida de las personas y el ciclo de desarrollo de las familias.

En esta misma línea es claro que, generalmente, las áreas receptoras de migrantes son dinámicas en términos económicos y concentran la inversión pública y privada, en tanto que las áreas expulsoras se encuentran estancadas y pierden a la población joven y mejor instruida. Como resultado, los ingresos promedio de los migrantes son mayores que los de los no migrantes (CEPAL, 2007: 633), y los hogares con migrantes tienen mayores ingresos mensuales que los hogares sin migrantes. La migración, a su vez, se ha facilitado por la reducción en los costos de transporte y tiempo de traslados.

La migración, en suma, se traduce, ya sea en un aumento o en una reducción de las desigualdades regionales, en función de los volúmenes de población implicada y de sus características. De acuerdo con los volúmenes se tiende a la convergencia regional; en tanto que con base en los atributos de la población ocurre una selectividad diferenciada según la variable del caso, lo que se traduce en divergencia. Asimismo, aunque la búsqueda de mejores condiciones de vida sigue siendo una de las principales motivaciones para la migración, éstas, de acuerdo con Rodríguez

\footnotetext{
${ }^{2}$ En el documento Panorama Social de América Latina 2007 de la CEPAL, se reporta que la selectividad femenina aplica en el caso de la migración campo-ciudad, en tanto que en los flujos ciudadciudad los resultados son variables de país a país. En el caso de México se ha encontrado que en lo relativo al nivel educativo más bien hay un intercambio interregional.
} 
(2008: 144), pueden en ciertos momentos o circunstancias estar desvinculadas del crecimiento económico y de los mercados laborales.

Con respecto a los factores relativamente emergentes que provocan la migración, se reconocen los efectos del grado de madurez de los sistemas urbanos en su actual etapa metropolitana, caracterizada por una notable movilidad intraurbana e intermetropolitana, debida a la expansión física de las ciudades y la formación de vínculos funcionales por causas laborales (empleo e ingreso), pero también por cuestiones de residencia. En otros términos, se reitera que la migración no es sólo por motivos económicos sino también puede ser por motivos de estudio, de salud, por violencia y por cuestiones familiares (Granados, 2007).

Producto del continuo proceso de urbanización en América Latina, la migración entre ciudades desplazó desde el último cuarto del siglo $\mathrm{xx}$ a la migración rural urbana. Ello, aunado a la desconcentración demográfica en los sistemas de ciudades, produjo que la migración dejara de tener el patrón concentrador característico de épocas pasadas. Las ciudades principales de prácticamente todos los países con alto grado de urbanización han sido consideradas como expulsoras netas de migrantes hacia las ciudades secundarias más dinámicas, arguyendo como causa, los efectos negativos que la crisis del modelo de producción por sustitución de importaciones tuvo en su base económica a fines del siglo pasado. Una peculiaridad de este tipo de migración es que en gran parte ha ocurrido hacia las ciudades del entorno inmediato de la ciudad primada, lo que ha configurado sistemas de ciudades policéntricos. Sumado a esto, la migración intrametropolitana hacia la periferia de las ciudades amplía la extensión territorial de las grandes urbes generando procesos complejos de reconfiguración territorial y funcional. En consecuencia, hay dudas respecto a si es del todo acertado considerar a las grandes ciudades como expulsoras netas de población ya que también continúan recibiendo altos volúmenes de personas, en tanto que la población saliente se dirige al entorno inmediato, ${ }^{3}$ por lo que se puede estar hablando de una expulsión ficticia que más bien es parte de la expansión metropolitana (Aroca, 2004; Guzmán et. al., 2006). Esto correspondería a un proceso de desconcentración-concentrada (UNFPA, 2007; Henderson, 2000; CEPAL, 2007; Pinto da Cunha, 2002; Rodríguez, 2002; Garza, 1999).

En este sentido, se trata de la consolidación de una forma de organización territorial de urbanización extensiva e intensos vínculos funcionales que articulan los mercados de trabajo regionales con las economías de escala y de urbanización, que a su vez, estimulan una determinada locali-

\footnotetext{
${ }^{3}$ Parte de la población cambia su lugar de residencia pero no su lugar de trabajo, lo que da lugar a una movilidad cotidiana o fenómeno de commuting.
} 
zación de las actividades productivas y se acompañan de ciertos tipos de ocupación residencial. Desde esta perspectiva, también se reconoce en la base económica de las ciudades y sus cambios, un factor adicional determinante en la orientación y monto de los flujos de migración interurbana.

Lo descrito tiene que ver, asimismo, con el planteamiento de los ciclos urbanos o ciclos de movilidad de la población, vinculados a los ciclos de vida de las familias ya mencionados y que se traducen en diferentes patrones residenciales al interior de los espacios metropolitanos. Dado que la urbanización campo-ciudad ha perdido importancia, son el crecimiento natural de la población y los patrones de movilidad residencial por proximidad, junto con la evolución de los mercados de trabajo, los que se traducen en las actuales formas urbano-regionales de la expansión urbana. Una de las aportaciones más importantes en este sentido a nivel internacional, y que tuvo eco en México, es el modelo de urbanización diferencial de Geyer y Kontuly (1993), quienes explican diversas modalidades migratorias con base en el ciclo de desarrollo urbano.

Para estos autores, en un sistema urbano, las ciudades grandes, intermedias y pequeñas experimentan ciclos de crecimiento rápido y lento, que expresan, primero, una tendencia a la concentración, y posteriormente, a la dispersión o concentración, a lo cual denominan urbanización diferencial. En cada ciclo predomina un tipo de flujo migratorio principal, acompañado de flujos secundarios, que significan, a su vez, el predominio ya sea de fuerzas concentradoras o de fuerzas desconcentradoras, mismas que modelan al sistema urbano. Este modelo explicativo, sin embargo, ha tenido un mayor impacto en los estudios urbanos que en los que se constrińen al análisis de la migración interna, como se concluye de los estudios realizados en nuestro país.

\subsection{Estudios en México}

En México las investigaciones recientes sobre migración interna a nivel nacional y en la región Centro pueden agruparse en las categorías de investigaciones que enfatizan aspectos principalmente demográficos de la migración en el sentido de su dinámica, dirección y volumen; en aquellos que la vinculan con la urbanización y las tendencias de concentración y desconcentración en el largo plazo, incluidos los distintos enfoques sobre ciclos urbanos, y los que la relacionan con el dinamismo económico y con la evolución de los mercados de trabajo. Sus hallazgos se han influido de forma recíproca y son parte de un cuerpo analítico sólido con aporta- 
ciones metodológicas, que en conjunto permiten entender las causas y los efectos de la migración interna en el centro del país. ${ }^{4}$

Entre los estudios ubicados en la primera categoría, Negrete (1990), al examinar el proceso migratorio hacia la ciudad de México, identifica tres procesos en ese momento poco estudiados por su novedad: 1) la reducción progresiva del número de inmigrantes a la ciudad; 2) el arribo de población con una baja calificación para el empleo, y 3) un reajuste del patrón migratorio que implicó un incremento de emigrantes desde la ciudad de México hacia las ciudades circunvecinas a ésta.

En otro análisis, Negrete (1999) clasifica a los municipios con base en su saldo neto migratorio en tres tipos: atractores, de rechazo y en equilibrio. Con este ejercicio, reconoce un proceso de desconcentración territorial de los migrantes desde la ciudad de México hacia su periferia conurbada y la corona de ciudades que la rodean, determinado por la pérdida de capacidad concentradora de la ciudad de México (que impacta su ritmo de crecimiento), y la reorientación de las corrientes migratorias, influenciadas por la activación económica de otros núcleos urbanos al interior de la misma región Centro. Estos hallazgos coinciden con los de Corona y Luque (1992).

Para Chávez y Savenberg (1995), entre los elementos distintivos de este nuevo horizonte migratorio sobresale su carácter diferencial con base en el sexo, la edad y las razones por las que migran las personas, así como por su alcance geográfico. Por ejemplo, los jóvenes vislumbran como destino a las grandes urbes con miras a buscar empleo, mientras que los adultos y su familia buscan las periferias de las ciudades para establecer su residencia, pero conservan su empleo en el centro. Este patrón migratorio tiene repercusiones en la reconfiguración sociodemográfica y espacial de los núcleos urbanos del centro del país.

Con un enfoque en las diferentes formas de movilidad de la población con origen en la Zona Metropolitana de la Ciudad de México (zMCM) y sus alcances geográficos, Graizbord y Mina (1994) concluyen que la región Centro representa para sí misma, la principal abastecedora de inmigrantes, lo que les lleva a reconocer un proceso inmigratorio de impacto megalopolitano.

Con respecto a los estudios de la segunda categoría sobre migración, urbanización y tendencias de la concentración y desconcentración en el

\footnotetext{
${ }^{4}$ Todas estas investigaciones, de hecho, tienen como punto de partida en su argumentación los cambios ocurridos durante los años ochenta en México y Latinoamérica en la dinámica de la urbanización, pero también los cambios observados en países del primer mundo en los ańos setenta. Reconocen en general una nueva etapa en la dinámica demográfica y en el proceso de urbanización del país a partir de esos años, que se tradujo de manera muy visible en la reducción del ritmo de crecimiento urbano de las grandes ciudades, lo cual se superpuso con una grave crisis económica y la transición de entre dos modelos de desarrollo económico.
} 
largo plazo, Graizbord y Mina (1994), así como Ruiz (1999) señalan que las pautas concentradoras de la población en el centro de México se tornaron difusas desde los años ochenta y estaban llegando a un punto de inflexión, lo cual implicaría una reversión de las fuerzas centrípetas a favor de una desconcentración hacia las ciudades de tamaño intermedio. Sostienen que este proceso abarcará tarde o temprano a la totalidad del Sistema Urbano Nacional y todas sus regiones, aún cuando no suceda con la misma fuerza en todas partes. Esta hipótesis tiene sus antecedentes en el trabajo pionero de Graizbord (1984) en el que, con un enfoque ex-ante, considera que el futuro de la urbanización sería de mayor equilibrio en la distribución territorial de la población y las actividades económicas.

En oposición, para Garza (1990), sin medidas correctivas, la concentración en las grandes urbes tendería hacia una superconcentración espacial, y el crecimiento de otras ciudades no sería a costa de la disminución del tamaño de las grandes ciudades (Camas, 1999). Más adelante Garza (1999) señala que es necesario analizar los procesos desde otra escala ya que más que un proceso de descentralización, lo que ocurre es un cambio en el ámbito de concentración, hacia conglomerados megalopolitanos y regiones urbanas policéntricas. Ambos autores suponen implícitamente que la migración ocurre desde las áreas estancadas hacia las dinámicas.

Otro tipo de aproximaciones enfatizan el grado de madurez de los sistemas metropolitanos. Así, desde el gobierno federal (Conapo, 2002) se realizó un estudio sobre las tendencias de la migración en las grandes metrópolis del país con base en el modelo de urbanización diferencial de Geyer y Kontuly (1993); en éste se compara la dirección y flujo de los movimientos migratorios para los años 1987-1992 y 1992-1997 con base en las encuestas ENADID 1992 y 1997 para las tres ciudades principales del país, además de Toluca, Torreón, Puebla y el resto del país. Se concluye que entre los dos periodos estudiados ocurrieron cambios profundos en los movimientos migratorios de las zonas metropolitanas, y que la transición urbana y de movilidad es más avanzado en las ciudades grandes. La ZMCM, por ejemplo, reduce los movimientos de su población en todas direcciones, predominan los flujos que se originan en su contorno central con destino a la periferia o resto del país, y más recientemente aumentan su importancia los movimientos desde las áreas suburbanas hacia el resto del país. Se prevé que en el futuro continúe la desaceleración del crecimiento demográfico de las ciudades más grandes, por lo que habría un patrón desconcentrador más maduro con movimientos en un espacio jerárquico no necesariamente continuo.

Entre los estudios del tercer tipo que se han ocupado de identificar las relaciones entre economía y migración en la región Centro de México, o que han ahondado en el análisis particular de alguna de estas variables, 
se considera que los espacios con mayor dinamismo económico constituyen zonas de atracción de corrientes migratorias, ya que configuran un ambiente laboral propicio.

Chávez y Guadarrama (2000 y 2004) llaman la atención sobre la transformación del mapa migratorio al interior de la región Centro, por cambios de las zonas de expulsión y atracción como efecto de: 1) la desconcentración industrial hacia algunas áreas urbanas periféricas a la Zona Metropolitana de la Ciudad de México (ZMCM), y 2) una mayor articulación funcional de los sistemas productivos junto con la difusión del sector terciario. Procesos que, señalan, ocurrieron bajo un ambiente de crisis y reestructuración económica, que el país en general y la región Centro en particular, registraron desde los años setenta hasta la última década del siglo xx. También se refieren a la inestabilidad del crecimiento económico en los diferentes estados y municipios de la región, la cual tiene una relación evidente con los flujos migratorios en unos espacios, pero no en otros; en este sentido sugieren considerar las escalas históricas y territoriales en las que se sitúan esas relaciones.

Granados (2007), por su parte, estudia el impacto de la dinámica migratoria en el crecimiento demográfico de Pachuca como ciudad secundaria receptora de migrantes, así como la relación entre el aumento de la población y el comportamiento de los sectores productivos de la ciudad. Incluye en su análisis algunas características socioeconómicas de los migrantes y se propone ahondar en los efectos que la presencia de éstos acarrea en los lugares de destino. Se apoya en la posibilidad que permitió el Censo de Población del 2000 para identificar a los individuos que residiendo en un municipio trabajan en otro, y de esa manera distinguir entre migrantes interestatales e intraestales. Encuentra que en la migración interestatal predomina la femenina y familiar y, que en la más reciente, los niveles de educación son mayores y tienen más altos ingresos que la población no migrante; en el caso de los migrantes intraestatales recientes se trata también de personas más jóvenes, recibe mayores ingresos y, como los primeros, se asientan en zonas no marginadas.

Como se aprecia, cada uno de estos análisis es un valioso aporte para entender la compleja relación que se establece entre dinámica económica y migración, y son asimismo, las referencias que contribuyen a plantear la hipótesis central de esta investigación, ya mencionada en la introducción. En las páginas siguientes, previo señalamiento del procedimiento metodológico, se revisa, a escala municipal y al mismo tiempo de metrópolis, la distribución y el dinamismo de los tres grandes sectores económicos en la región, así como su relación con la orientación final de los flujos de inmigración reciente de acuerdo al género y la edad de los migrantes. 


\section{Delimitación de la región y procedimiento metodológico}

La región Centro la integran el Distrito Federal y los estados de Hidalgo, México, Morelos, Puebla, Querétaro y Tlaxcala, cuya superficie total abarca $97,964 \mathrm{Km}^{2}$, equivalente a $4.9 \%$ de la superficie total del país. No obstante representar una reducida fracción del territorio nacional, su población en 2005 ascendió a 34.7 millones de habitantes $(33.6 \%$ del total nacional) con una densidad poblacional aproximada de 355 habitantes $/ \mathrm{Km}^{2}$.

En materia económica, la región Centro genera, según datos de los censos económicos de 1999 y 2004 utilizados para este análisis, 42.1\% del PIB total nacional; $16.3 \%$ del PIB del sector primario; $44.6 \%$ del PIB manufacturero y $43.5 \%$ del PIB terciario. Estas cifras demuestran que, a pesar de los efectos de la crisis económica de los años ochenta y la reestructuración económica que experimentó durante los noventa, la región, en general, y la ZMCM, en particular, (como núcleo de la región), continúan siendo las principales concentraciones demográficas y económicas del país, si bien la concentración económica es mayor que la demográfica. Ambos espacios, sin embargo, han reducido su aportación al PIB total nacional desde los ańos ochenta, al cambiar de $43.5 \%$ en 1980, a $41.2 \%$ en 1998, y 40.3\% en 2003, en el caso de la región Centro. En el caso de las dos entidades en donde queda comprendida la ZMCM que son el Distrito Federal y el Estado de México, su participación conjunta ha disminuido para esos mismos años de $37.4 \%$ en 1980 para mantenerse en $32.7 \%$ en 1998 y 2003.

El procedimiento metodológico seguido para el análisis económicoregional requirió la construcción de una base de datos a partir de los Censos Económicos de 1999 y 2004. Se utilizó la variable de valor agregado censal bruto (VACB) por gran sector de actividad, para los 553 muni$\operatorname{cipios}^{5}$ y las 12 zonas metropolitanas oficiales que integran la región Centro $^{6}$ (Sedesol, 2004; Conapo, 2002; INEgI, 2004). En el caso del vaCB se realizó un ajuste con base en el pIB del Sistema de Cuentas Nacionales, con el propósito de que los datos censales reflejen adecuadamente las magnitudes macroeconómicas. El resultado puede considerarse como PIB, mismo que se convirtió a pesos de 1993, pensando sobre todo en incorporar más adelante, el análisis de censos económicos de años previos. Por último, se obtuvieron rangos para la distribución del PIB entre los municipios con base en la desviación estándar, y los resultados se presentan cartográficamente para su mejor ilustración.

\footnotetext{
${ }^{5}$ El conteo de unidades político-administrativas es el siguiente: Distrito Federal 16 delegaciones; municipios: Hidalgo 84; México 125; Morelos 33; Puebla 217; Querétaro 18, y Tlaxcala 60.

${ }^{6}$ Ver cuadro A.1 del anexo.
} 
El periodo de análisis corresponde al final de los años noventa y principios del primer lustro del siglo xxI (1998-2003 para datos económicos y 2000-2005 para datos demográficos), que es un periodo de transición entre la recuperación económica de los años noventa posterior a la década perdida y la recesión con que comienza el primer decenio del 2000, vinculada a su vez a la recesión estadounidense de esos mismos años. Así, el ritmo de crecimiento de 3.0\% promedio anual del pIB nacional entre 1993 y 1998, se redujo a 2.5\% entre 1998 y 2003; sin embargo, la evolución del pIB fue muy contrastante en este último lustro al pasar de 5.2\% entre 1998 y 2000 a $0.7 \%$ entre 2000 y 2003 . El periodo de estudio, en suma, corresponde a un periodo de recesión económica nacional y regional.

\section{Dinámica económica sectorial en la región centro}

\subsection{Sector primario: declive y reestructuración territorial bajo un ambiente recesivo}

En primer término es importante aclarar que en el sector primario únicamente se consideran las actividades de pesca y acuicultura animal. Esto se debe a que no existe un censo agropecuario actualizado; por consiguiente, se concede la observación de que estas dos actividades no son representativas al cien por ciento del sector agropecuario. Empero, sí se asumen como actividades que dentro de ese sector son de alto valor comercial y por tanto de alta productividad; de ahí su marcada concentración territorial, como se mostrará a continuación.

Los resultados del análisis de este sector de actividad en la región Centro muestran, como rasgo principal, su dinamismo negativo. Así, el Рів primario se redujo de 19.4 mil millones de pesos en 1998 a 14.3 mil millones en 2003, con una Tasa de Crecimiento Promedio Anual (TCPA) de -6.0\%; en segundo lugar, de los 12 los municipios ${ }^{7}$ de los rangos uno y dos que en 1998 concentraban $82.6 \%$ del PIB primario y $14.1 \%$ de la población de la región Centro en 2000, un lustro después, si bien el número de los municipios más importantes se mantiene casi constante (11), su participación en el PIB se reduce a $58.8 \%$ y en la población cae a sólo $1.9 \%$ (cuadro 1). Esto habla de una gran variabilidad en el comportamiento del pIB primario (de tipo comercial) en los diferentes municipios a lo largo del quinquenio, lo que significa que no existen las condiciones para un desarrollo estable en el largo plazo.

\footnotetext{
${ }^{7}$ Estos 12 municipios corresponden a los comprendidos en los dos primeros rangos de la clasificación de municipios de acuerdo al рів que aportan al sector primario. De ellos, nueve aportan un PIB que va de 430,000 a 2.1 millones de pesos de 1993 (rango uno), y tres aportan un PIB de 295,000 a 430,000 pesos (rango dos). En total, se obtuvieron siete rangos que se aprecian en el cuadro 1 .
} 


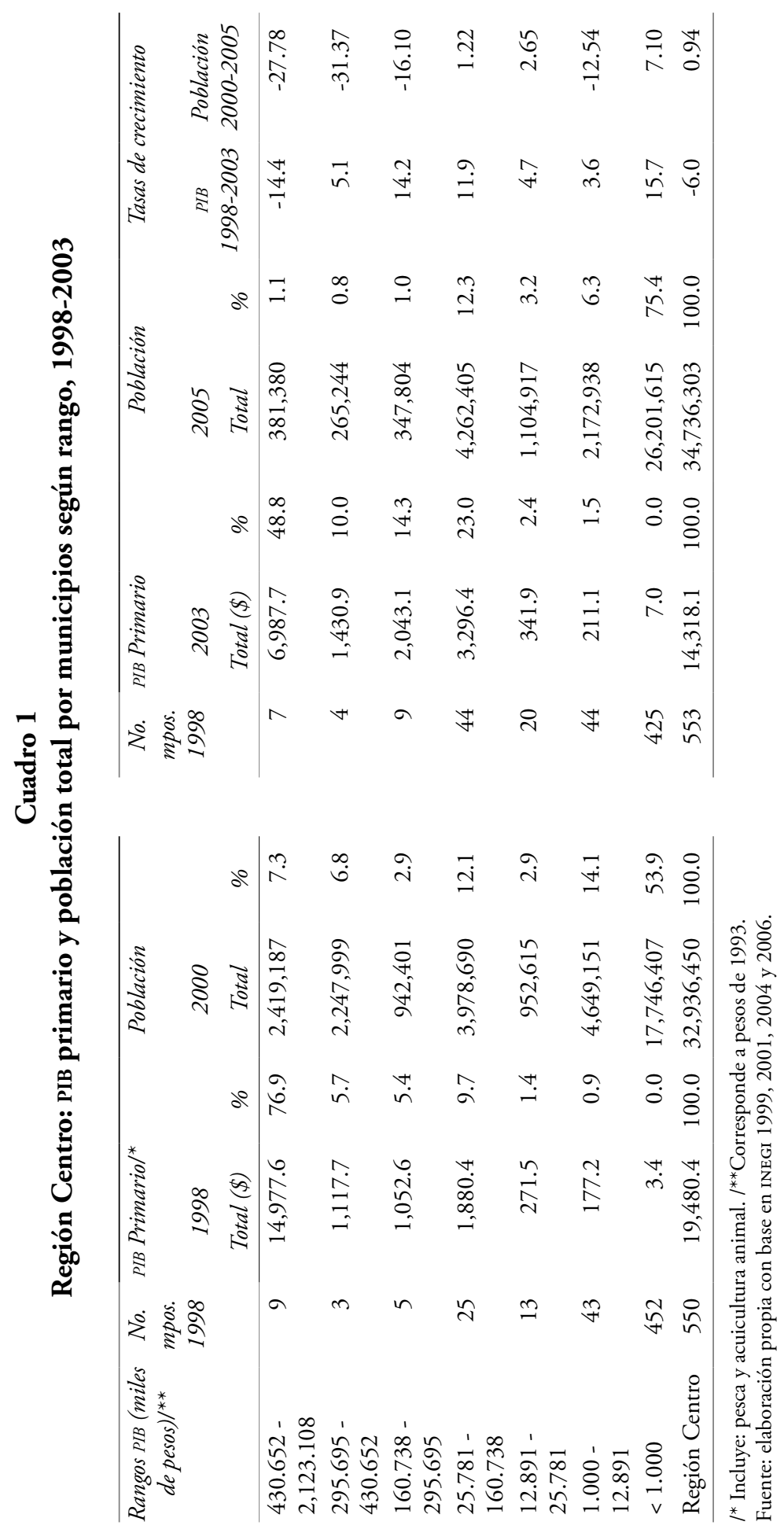


Visto a mayor detalle, el impacto negativo del decrecimiento del PIB ocurrió en el rango uno de los municipios que hacían la mayor aportación al PIB en el año inicial (76.9\%) y que estaban ligados a economías de aglomeración dada su población de 2.4 millones de habitantes o $7.3 \%$ del total regional (cuadro 2 y descripción del mapa I). Si se consideran los municipios de los cuatro primeros rangos, que eran un total de 42, éstos contribuían con $97.7 \%$ del pIB y $29.1 \%$ de la población.

Para el año final, la actividad primaria se dispersó un poco más, al ser 64 los municipios que aportaban $96.1 \%$ del PIB, concentrándose ahora en los de rango tres y cuatro, sobre todo los segundos, cuya población total era de 4.2 millones de habitantes. Esto significa que algunos son los mismos municipios que en el año inicial ocupaban el rango uno, pero redujeron su capacidad de generación de PIB en el sector primario, y fueron reemplazados en esa posición por otros municipios también vinculados a economías de aglomeración pero de carácter periurbano (descripción del mapa II).

En contraparte, los municipios de rango siete ( 452 del total) en el año inicial sólo aportaron 3.4\% del PIв agrícola, pero $53.9 \%$ de la población. Para el ańo final fueron 425 los municipios ubicados en ese rango, que produjeron $7.0 \%$ del pIв y aumentaron su participación en la población total a 75.4 por ciento. Esto indica un aumento de la productividad del sector, pero al mismo tiempo que el avance de la urbanización en ellos ha sido continuo al tratarse de municipios cada vez más poblados, ubicados tanto en zonas metropolitanas (ZMCM) como en las periferias perimetropolitanas expandidas (alrededor de la zM de Querétaro, zMCM, zM de Toluca, zM de Apizaco, zM de Puebla-Tlaxcala, zM de Cuernavaca y zM de Cuautla). El resto de municipios en este rango tienen un carácter rural, sobresaliendo en número los de los estados de Puebla, México e Hidalgo, principalmente.

Los datos hasta aquí señalados permiten hacer dos importantes inferencias: 1) Como resultado del alto grado de urbanización de la región Centro y su vocación económica orientada a los sectores secundario y principalmente terciario, la actividad primaria tiene un comportamiento negativo; en su interior, sin embargo, su evolución es ambivalente pues resulta negativa en los municipios metropolitanos más urbanizados, pero es positiva en los municipios perimetropolitanos y sólo en algunos metropolitanos. 2) En consecuencia, y por esta ubicación territorial señalada, los municipios de menor participación en el PIB primario aumentan su población tanto en términos absolutos como relativos si corresponden a demarcaciones perimetropolitanas (o incluso metropolitanas), y la disminuyen si son rurales. Los municipios con mayor participación en el PIB primario en el año final, mientras tanto, disminuyen en términos relativos 


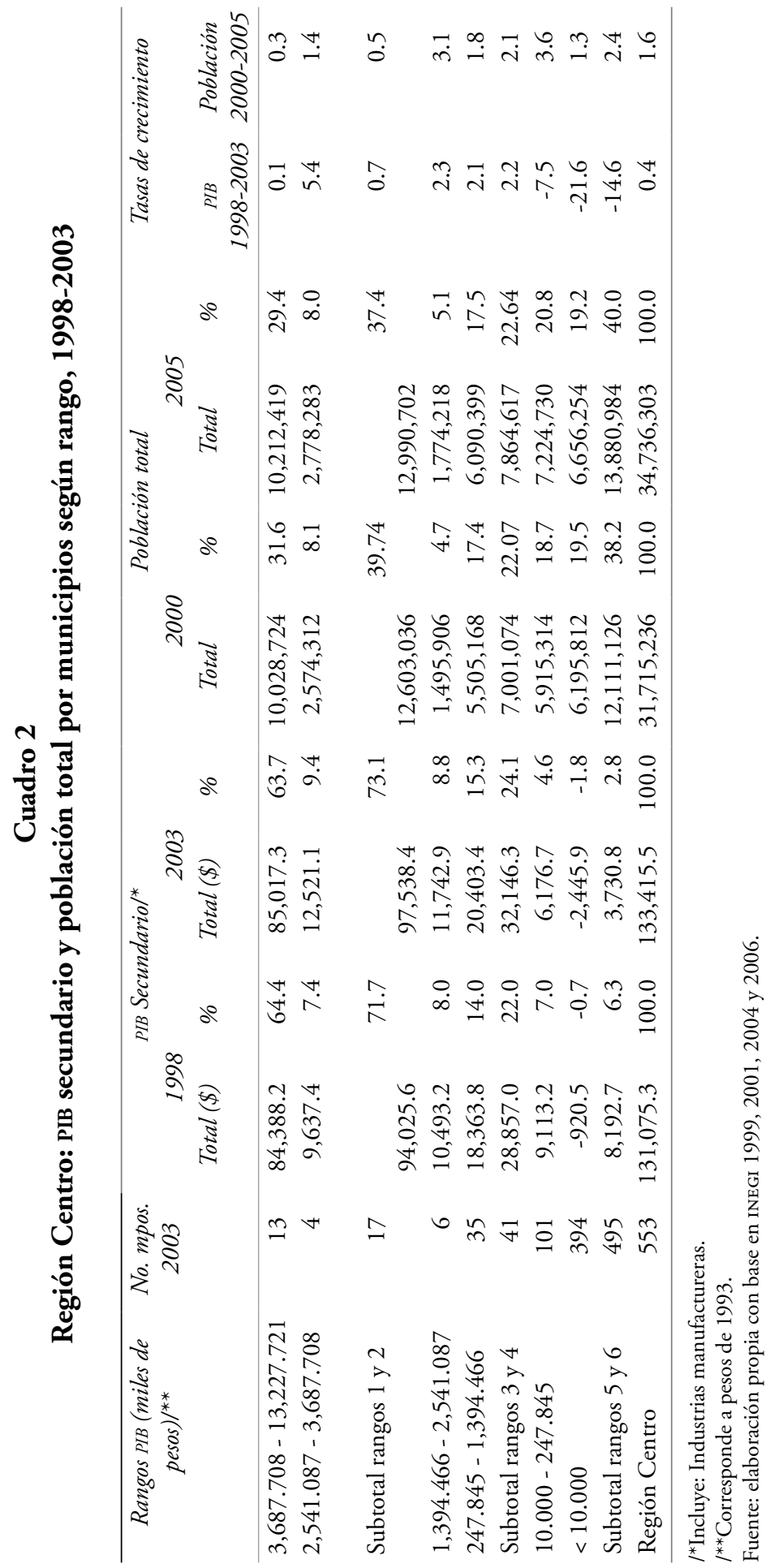




\section{Mapa I}

Región Centro: distribución territorial del PIB primario, 1998

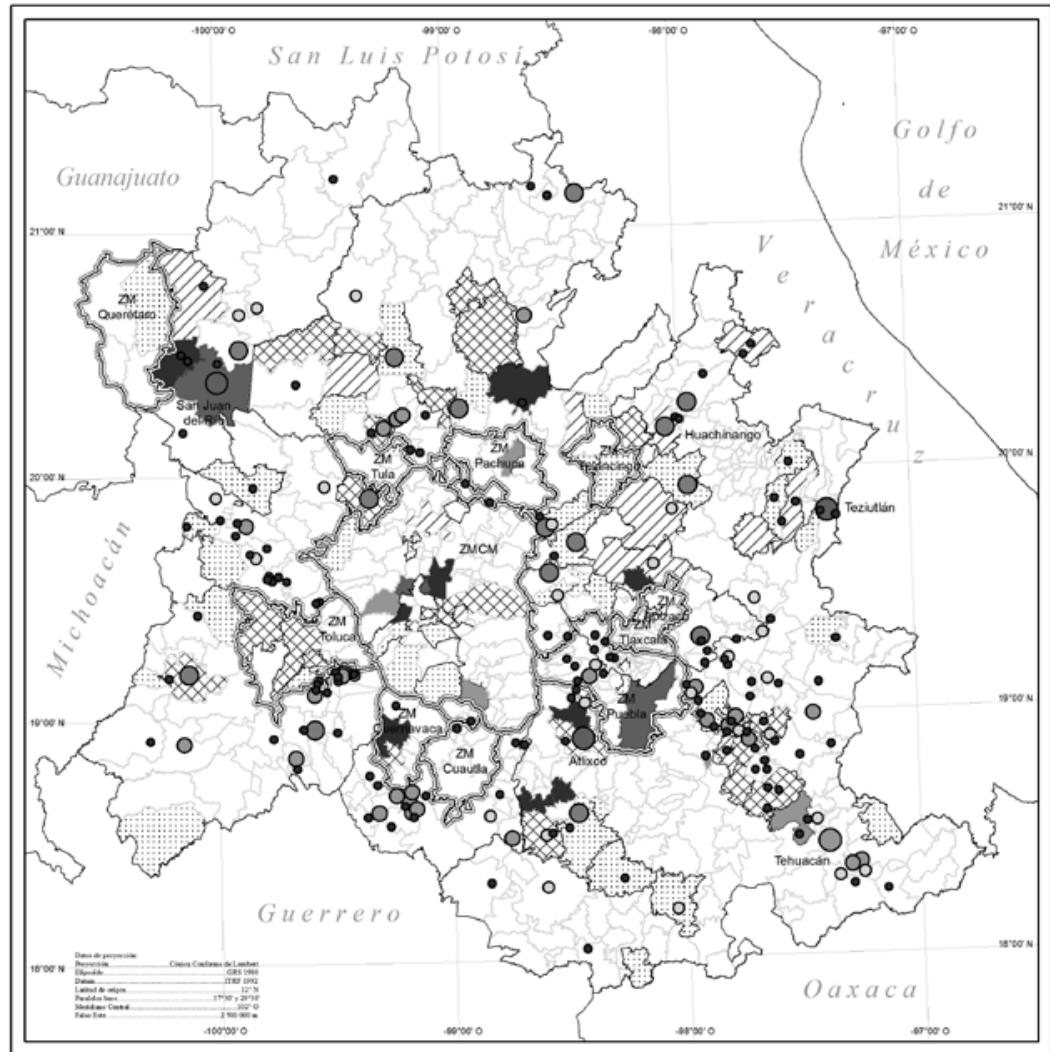

Millones de pesos

a precios constantes de 1993

$>430,1$ población-

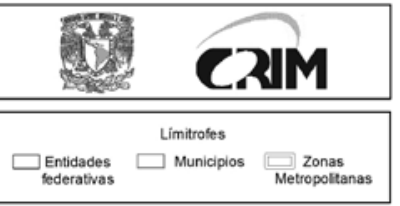

$160,1-300,0$

$26,1-160,0$

VD $13,1 \cdot 26.0$

1.0-13,0

100000 - 250000

50000 - 96999 (3)

$25000-49999$ (15)

O $15000-24999$ (28)

O $10000 \cdot 14999$ (27)

- $5000-9999 \quad(126)$

Escala apróximada: 1:2380000 kibmetros

$$
\text { (1) }
$$

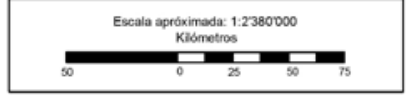

Fuente: Elaboración propia con base en INEGI (1999; 2000); (Sedesol, 2004; Conapo, 2002; INEGI, 2004). 


\section{Mapa II}

Región Centro: distribución territorial del PIB primario, 2003

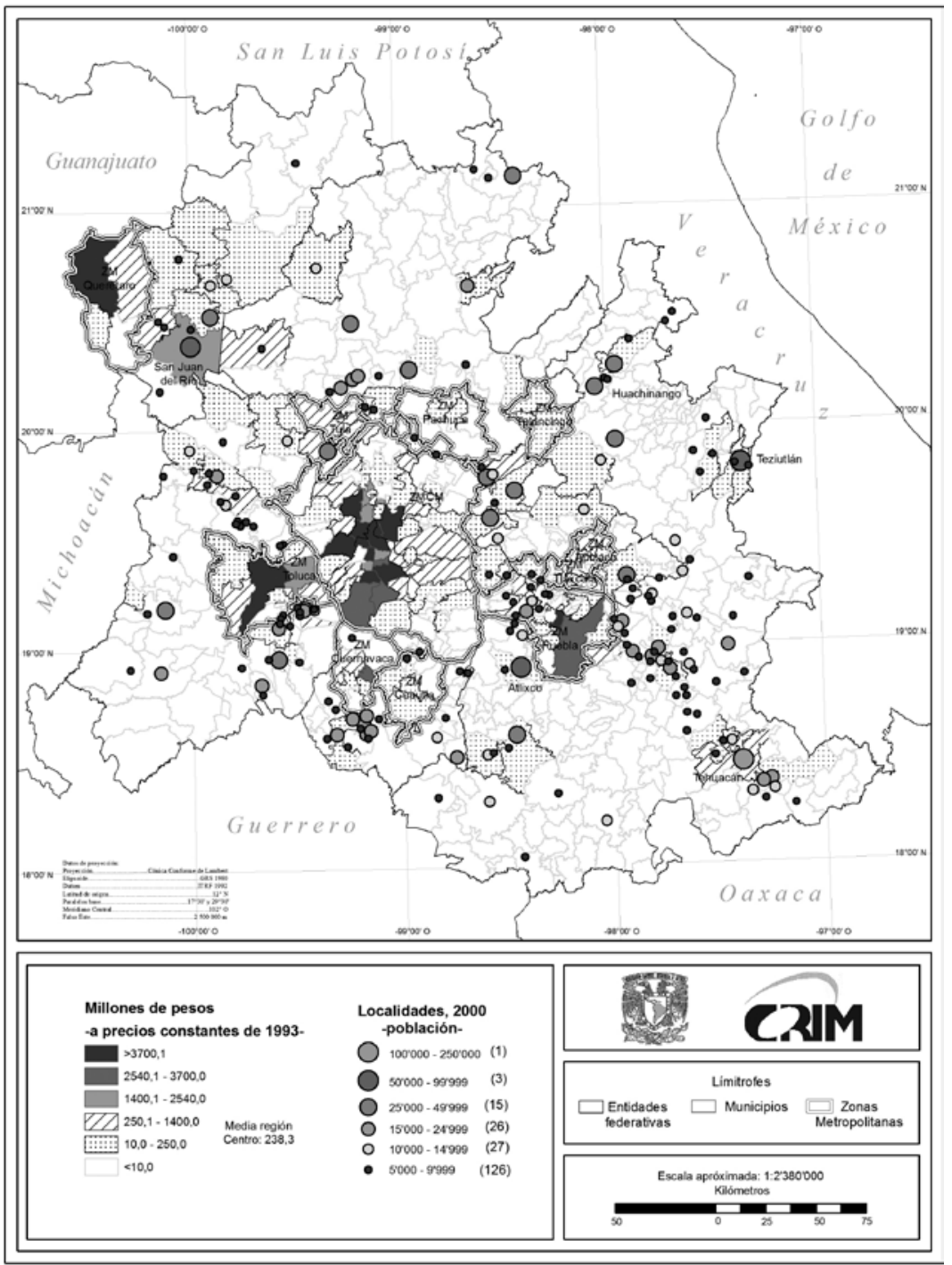

Fuente: Elaboración propia con base en INEGI (2004; 2005); (Sedesol, 2004; Conapo, 2002; INEGI, 2004). 
su participación en la población total, si bien continúan incrementando su población absoluta. El diferencial de población se estaría desplazando (presumiblemente), hacia los municipios económicamente más dinámicos al interior de la región (especializados en el sector secundario o terciario). Esta pérdida de población aplica independientemente de si la tasa de crecimiento promedio anual del PIB es positiva o negativa, ya que en ambas situaciones la productividad del sector primario se mantiene a la alza como resultado de que las tasas de crecimiento de la población son siempre menores que las tasas de crecimiento del PIB en todos los rangos.

Es conocido en la literatura sobre geografía económica y regional que el incremento de la productividad agrícola o del sector primario en general -entendido ello como el aumento de valor agregado por trabajador-, combinado con el cambio en las estructuras de consumo de la población, da como resultado una disminución del empleo en ese sector respecto al empleo total de un país o región. De esa manera, la población desplazada de esa actividad se suma a los sectores de la economía urbana, con lo cual se refuerza el proceso de urbanización (Polèse, 1998).

Ahora bien, al identificar con precisión los municipios que tuvieron mayor participación en la formación del PIB primario durante el periodo analizado, el rasgo más sobresaliente es su inestabilidad, ya que sólo dos casos se repiten: Tianguismanalco en Puebla y Atlangatepec en Tlaxcala. Los restantes municipios no repiten en la lista. Esta inestabilidad puede deberse a que las actividades del sector primario, aquí consideradas, corresponden a aquellas de carácter comercial cuya demanda es muy dependiente de la estabilidad en el ingreso de la población que, como se sabe, está sujeta a los ciclos económicos, y en algunos casos inclusive, al ser productos de exportación como algunas hortalizas y flores, están sometidos a fluctuaciones en los precios internacionales, a la vez que enfrentan una fuerte competencia en el mercado mundial. Otro aspecto que se puede inferir del cuadro es que en algunos casos la producción primaria consiste en agricultura urbana o periurbana en pequeños espacios (fundamentalmente en viveros) en municipios metropolitanos, por lo que enfrentan la competencia de otras actividades más rentables que pueden pagar mayores precios por la renta de la tierra y provocan su desplazamiento.

Por otra parte, al comparar las tasas de crecimiento promedio anual (ТСРA) de la población de los municipios que concentran el pIB primario tanto en el año inicial como en el final, sólo algunos (rangos cuatro, cinco y siete) registran crecimiento demográfico por arriba del registrado por la región Centro (0.94) durante el quinquenio 2000-2005. En el caso exclusivo del rango siete, los municipios que lo integran tienen a la vez el mayor crecimiento económico en las actividades comerciales y de alta productividad del sector primario, y la mayor tasa de crecimiento de po- 
blación. Sin embargo, esto no significa que sean municipios atractores de población, sino que, al ser los de menor población total individualmente (menos de 40,000 habitantes en promedio), sus tasas de crecimiento demográfico suelen ser mayores. Esto tampoco descarta que sean receptores de flujos migratorios, si bien de muy poco peso, en caso de serlo, respecto del total de los flujos migratorios hacia la región.

En los mapas I y II se constata lo mencionado líneas arriba, en relación a que algunos de los municipios que en 1998 concentraban la mayor proporción del pIB primario, forman parte de alguna zona metropolitana (como las de la ciudad de México, de Puebla-Tlaxcala) o de su periferia. Alrededor de éstas se identifican también algunas tenues agrupaciones; por ejemplo, alrededor de San Juan del Río en Querétaro, y algunos corredores en formación, como los de Puebla-Tehuacán y Apizaco-Tulancingo. También se identifican en ese año zonas en donde el PIв primario no destaca a escala regional, como es el estado de Morelos, con la única excepción de Cuernavaca, que es a su vez el municipio que encabeza la lista de los territorios que mayor participación tienen en la formación del PIB primario en la región Centro en ese año.

Para 2003 -y manteniendo el valor de los rangos para hacer viable la comparación-, el patrón territorial cambia, pues salvo algunas excepciones, no hay coincidencia espacial de los municipios con mayor PIB primario, con los que forman parte de las zonas metropolitanas de la región (lo que es de esperarse dada la reorientación económica de la región hacia el sector terciario).

En esta ocasión los municipios de alto PIB primario son los ubicados en las áreas periurbanas circundantes a las zonas metropolitanas, donde ciudades pequeñas de menos de 50,000 habitantes constituyen el soporte material para perfilar la formación de agrupaciones subregionales al interior de la región.

Sobresale el caso particular de Morelos, que en el primer año del periodo -con excepción de Cuernavaca-no destacaba por su aportación al Рів primario, aunque para el 2003 presenta un boom productivo que territorialmente forma un corredor desde el sureste de la zM de Cuautla, hacia el oeste de la zM de Toluca (prologándose quizás fuera de la región, hacia Michoacán). Destaca también la alta concentración del PIв primario en los municipios de Querétaro, lo que se explica, en parte, por la función de esta entidad como territorio bisagra entre las regiones Centro y Occidente, particularmente con la zona del Bajío (Guanajuato y Michoacán) en donde la participación de las actividades primarias es importante en la formación del pIB.

En suma, el sector primario de carácter comercial en los municipios de la región Centro registró un declive en el periodo 1998-2003 que 
impactó principalmente a los municipios de carácter metropolitano, habiéndose, de esta manera, desplazado la actividad a otros municipios perimetropolitanos en los que encontró condiciones más propicias para su desarrollo, si bien con un impacto moderado en su crecimiento general. Los municipios beneficiados por la dispersión de la actividad primaria, por otra parte, no experimentaron un crecimiento demográfico importante. El crecimiento de población observado en el rango siete de los municipios en el año 2003 es sólo aparente, ya que se debió al cambio a ese rango que tuvieron municipios ubicados previamente en 1998 en los rangos uno y dos, principalmente, con alta población. Así, la actividad primaria no está asociada a un crecimiento demográfico.

\subsection{Sector secundario: estancamiento industrial y desconcentración concentrada}

El análisis del PIB industrial confirma la concentración del crecimiento económico en pocas unidades territoriales, ya que sólo 17 municipios (3.0\% del total de 553) en 2003 concentran $73.1 \%$ del pIB sectorial y $37.4 \%$ de la población de la región en 2005. El PIB manufacturero de esos municipios creció a una tasa promedio anual $0.74 \%$, la cual, aunque reducida, fue superior a la registrada por la región Centro en su conjunto (0.35) en ese mismo sector (cuadro 3). En otros términos, el pIB generado en los dos primeros rangos de municipios tuvo en el periodo 1998-2003 un desempeño industrial relativamente mejor al promedio regional (0.4\%); únicamente los municipios pertenecientes a los rangos tres y cuatro, por cuanto a su contribución en el PIB sectorial, tuvieron en conjunto un mayor dinamismo al crecer $2.2 \%$ anual, aunque su participación en el total de la región fue de $24 \%$ del pib en el año final (cuadro 2). Estos dos últimos rangos están integrados por 41 municipios y generaron casi la misma cantidad de PIB manufacturero regional durante el periodo que el generado en los municipios de rango uno y dos, y aumentaron en 863,000 habitantes su población o $28.6 \%$ del total (más del doble que los municipios de rango uno y dos). En el caso de los municipios de rango cinco y seis ocurrió una desindustrialización que implicó un decrecimiento de $-14.6 \%$ promedio anual.

La concentración de la industria en los municipios de los rangos uno y dos es más evidente con cifras absolutas, ya que durante el quinquenio 1998-2003 produjeron cada uno en promedio 206,000 millones de pesos de PIB. El promedio de los 41 municipios de rango tres y cuatro fue de 80,000 millones, y el de los 495 municipios de rango cinco y seis fue de 9,000 millones; la diferencia es bastante clara. Debido a esta situación, el РIв industrial en los 58 municipios comprendidos en los rangos uno-dos 


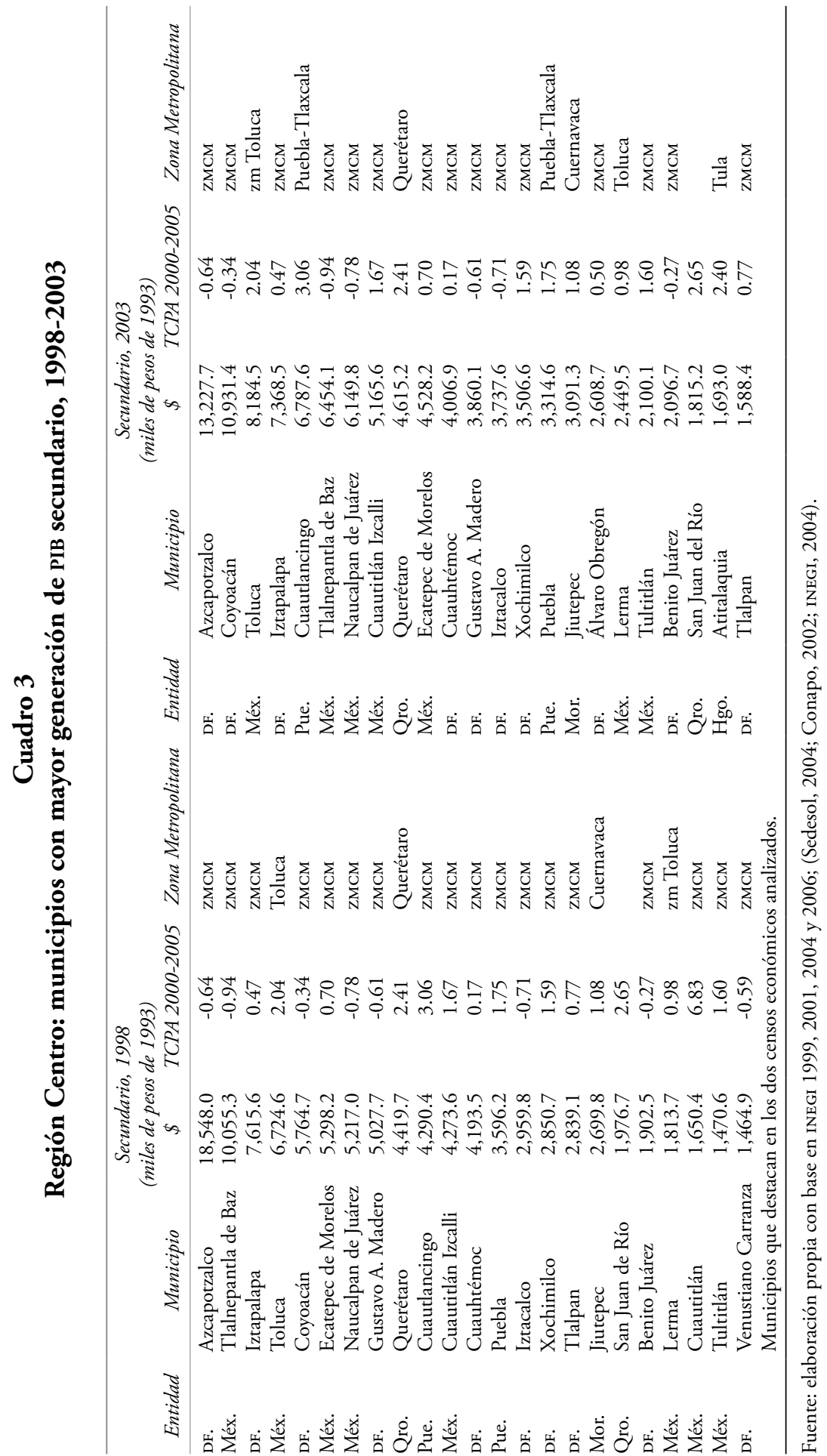


(17) y tres-cuatro (41) se incrementó de 93.7\% del total regional en 1998 a $97.2 \%$ en 2003; al mismo tiempo, sin embargo, se produjo una redistribución de su localización entre las zonas metropolitanas comprendidas (véase el apartado que sigue).

Con base en lo descrito, durante los años considerados ocurrieron dos procesos destacables: en primer término, un estancamiento del sector industrial en la región, con origen en los 13 municipios más industrializados del rango uno, pero principalmente en los menos industrializados de rango cinco y seis; en segundo lugar, se presentó una descentralización relativa de la actividad industrial que implicó únicamente a los municipios y zonas metropolitanas de los rangos uno al cuatro. Este proceso se aprecia sólo al observar los cambios en la distribución del pIB por zonas metropolitanas (cuadro A-1).

En lo que concierne a la población de los municipios de los dos primeros rangos por su contribución al PIB, ésta pasó de 12.6 a 12.9 millones de habitantes entre 2000 y 2005, lo que implicó un aumento de casi 387,000 personas con una TCPA de $0.5 \%$; en cada municipio la población aumentó 22,800 habitantes en promedio. En este sentido, el crecimiento de la manufactura en estos municipios estuvo ligado a un incremento de la población. De igual manera, el crecimiento del PIB en los municipios en los rangos tres y cuatro también se tradujo en crecimiento demográfico (2.1\% anual), lo que significó un aumento de 863,000 personas (o 21,000 habitantes por municipio), confirmando el vinculo positivo entre crecimiento económico y demográfico. En sentido opuesto, la población de los municipios de rango cinco y seis, si bien aumentó en 1.7 millones de personas con una TCPA de $2.1 \%$, el promedio de aumento de cada municipio fue de 3,500 personas. Las bajas tasas de crecimiento demográfico de los municipios más industrializados de la región son producto de sus elevados grados de urbanización y de su carácter metropolitano.

Una observación adicional es que la distribución de la población en la región no tiene el mismo grado de concentración que el PIB, y en todo caso es al contrario, o bien tiene una distribución más equilibrada. Así, por ejemplo, $40.0 \%$ de la población se ubica en los rangos cinco y seis de los municipios, mismos que sólo aportan $6.3 \%$ del PIB industrial regional; les siguieron los rangos uno y dos con $37.4 \%$ de la población y $71.7 \%$ del PIB; en una situación intermedia se ubican los rangos tres y cuatro con $22.6 \%$ de la población y $22 \%$ del PIB. Aunque el rango uno tiene por sí solo la participación más alta en la población total con $29.4 \%$ del total, lo cual se debe a su carácter metropolitano. 


\subsection{Expresión territorial}

Según se observa en el cuadro 3, que muestra los 23 municipios donde se concentra el sector manufacturero, éstos son los mismos en 1998 y 2003 en el caso del rango uno (13 de 13) y en el rango dos (4 de 4); en el caso del rango tres repiten 4 de 6 . En total, en los tres primeros rangos de municipios según PIB, sólo dos de los existentes en 1998 no repiten en 2003. Sobresale asimismo que, con excepción de San Juan del Río (Querétaro), todos los demás municipios forman parte de alguna zona metropolitana, con la ZMCM como el área de mayor concentración. De esta forma se ratifica la ubicación preferentemente urbana (en general) y metropolitana (en particular), del sector industrial.

La concentración del PIB secundario en la ZMCM continuó reduciéndose a favor de las otras zonas metropolitanas de la región al disminuir su participación de $73.3 \%$ del total en 1998 a 68.2\% en 2003. Como resultado, la zM de Toluca aumentó su participación de 8.3 a $10.3 \%$ en el mismo periodo; la zM de Puebla-Tlaxcala pasó de 7.7 a $8.9 \%$; la zM de Querétaro de 4.2 a $5.4 \%$ y la zM de Cuernavaca de 2.8 a $2.9 \%$ (cuadro A.1). Esta combinación de procesos confirma la tendencia a una relocalización intermetropolitana de la manufactura en la región Centro, de acuerdo con lo ya señalado en otras investigaciones (Olivera y Guadarrama, 2002).

Acorde con los procesos de redistribución y de concentración que indican los datos estadísticos, su traducción cartográfica permite constatar el atributo relativo del primer proceso y el carácter metropolitano del segundo, que de manera más apropiada deben caracterizarse como un proceso de desconcentración concentrada. En primera instancia, entre el mapa III (de 1998) y el mapa IV (de 2003) no se observan cambios importantes; las variaciones más significativas suceden en los municipios pertenecientes a los rangos de más baja creación de PIB, al detectarse un patrón territorial menos disperso en 2003. De esta manera, el PIB industrial, se concentra en el interior de las zonas metropolitanas, o bien, forma pequeńos corredores en detrimento de aquellos municipios alejados que en 1998, no obstante contar con alguna localidad de entre 15,000 y 50,000 habitantes, en el corto plazo no pudieron resistir los efectos de su aislamiento. El caso más representativo es el de Morelos, en donde el sector secundario, para el periodo señalado, tendió a concentrarse tanto en la Zona Metropolitana de Cuernavaca como en la de Cuautla, retirándose de la parte sur de la entidad.

Por otra parte, destaca que los municipios pertenecientes al rango cinco por su aportación al PIB industrial, que corresponden a los que tuvieron las mayores tasas de crecimiento demográfico, se localicen pre- 


\section{Mapa III \\ Región Centro: distribución territorial del PIB secundario, 1998}

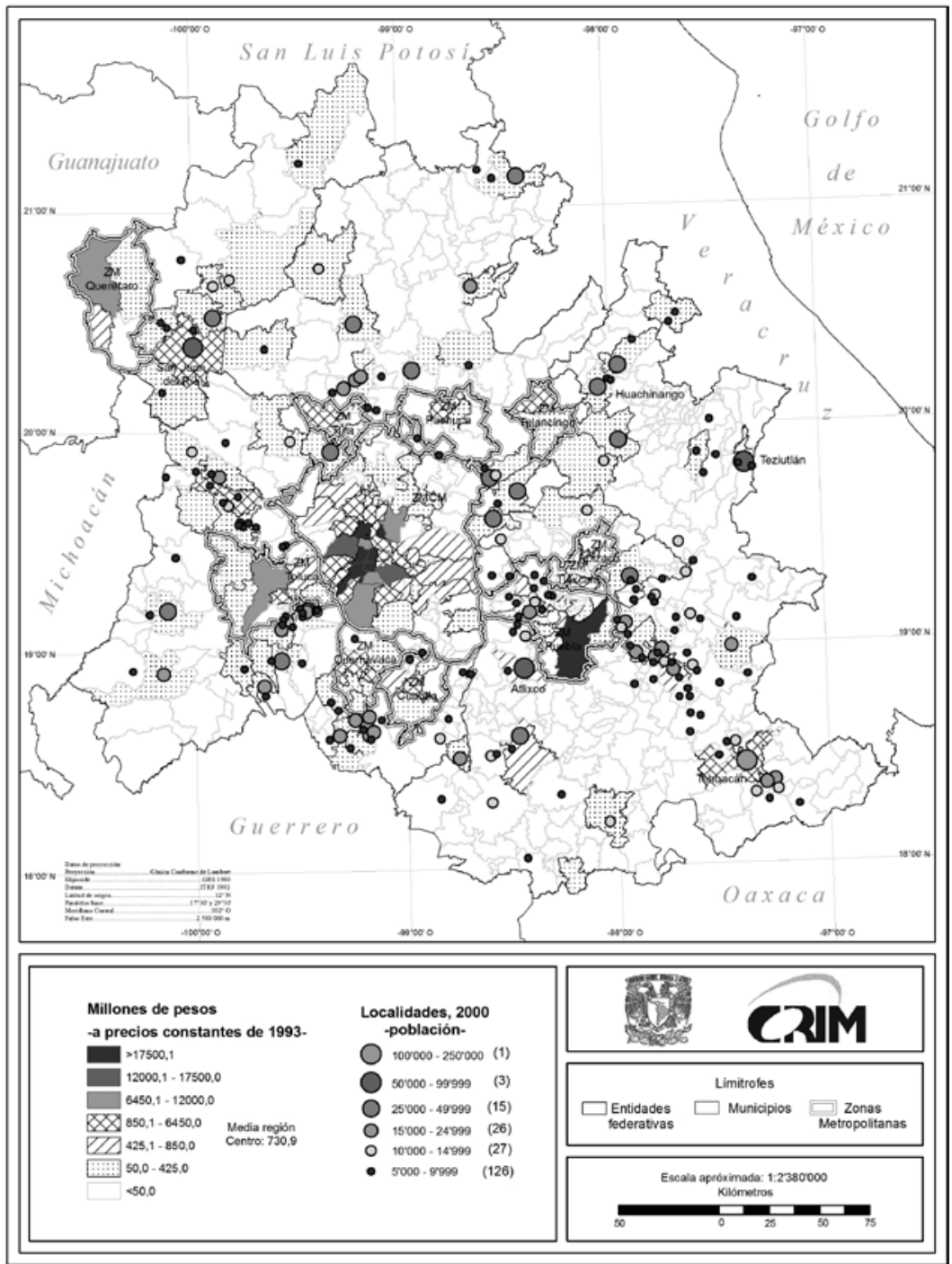

Fuente: Elaboración propia con base en INEgi (1999, 2000); (Sedesol, 2004; Conapo, 2002; INEGI, 2004). 


\section{Mapa IV}

Región Centro: distribución territorial del PIB secundario, 2003

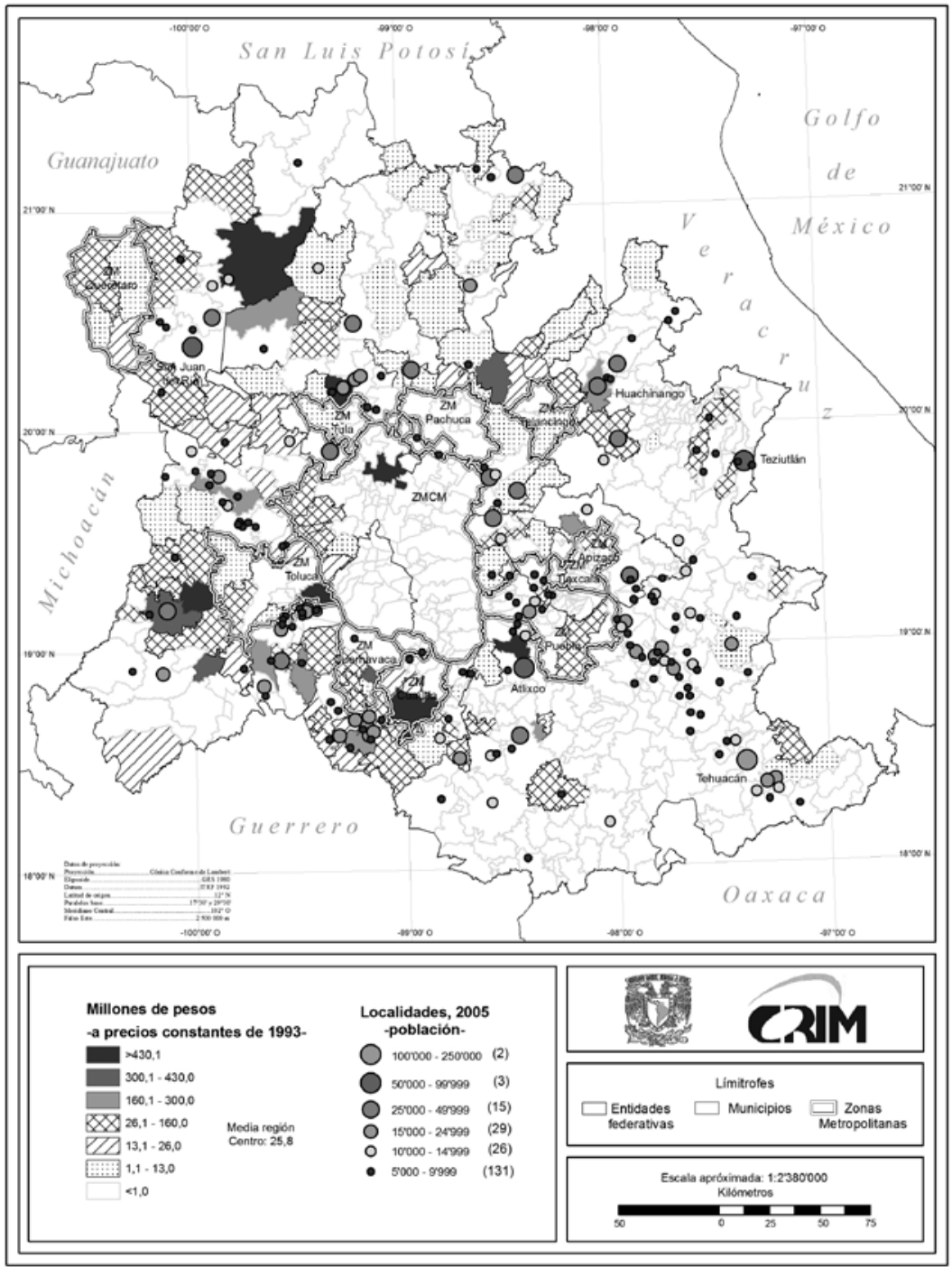

Fuente: Elaboración propia con base en INEGI (2004; 2005); (Sedesol, 2004; Conapo, 2002; INEGI, 2004). 
ferentemente en las periferias metropolitanas, coincidiendo con la ubicación de ciudades pequeñas (entre 5,000 y 50,000 habitantes), y formando un gran anillo alrededor de la ZMCM y de las Zonas Metropolitanas de Toluca, Puebla-Tlaxcala y Querétaro.

También sobresale por su participación en el PIB de este sector de actividad, la presencia, paralela a los ejes carreteros principales, de los municipios de San Juan del Río (Querétaro) y Tehuacán (Puebla). Lo que resulta indicativo del carácter policéntrico de la región Centro, proceso que se originó durante la crisis y reestructuración industrial registrada en la ZMCM en los ańos ochenta y noventa, y que se tradujo en una desconcentración concentrada de la manufactura en el ámbito de la región Centro. Como ya se refirió, la concentración territorial del PIB manufacturero ocurre en zonas metropolitanas, principalmente en las de mayor jerarquía $y$, al interior de éstas, en sus municipios centrales o del primer contorno de expansión, que son en los que históricamente se ha localizado la base productiva industrial.

Otro rasgo territorial que se reconoce con el análisis de este sector es la formación de corredores; por ejemplo, el que se forma a partir de la Zona Metropolitana de Toluca, para unirse hacia el norte con Atlacomulco (en el Estado de México) y un pequeño grupo de ciudades chicas, para posteriormente proyectarse hacia el Bajío. Otro corredor y quizás el más importante, es el que desde el noroeste de la región, en Querétaro (al parecer es una extensión del corredor del Bajío), articula a San Juan del Río, la ZM de Tula, se extiende hacia la ciudad de México para prolongarse hacia Puebla y posteriormente a Tehuacán (e incluso llegar hasta Oaxaca).

Por entidad, $74.4 \%$ del PIB manufacturero de la región Centro se concentra en el Distrito Federal y el Estado de México, 10.6\% en Puebla y 6.6\% en Querétaro. Las mayores tasas de crecimiento demográfico en el periodo 1990-2005, mientras tanto, corresponden al Estado de México y Querétaro, entidades en las que presumiblemente se relocalizó la actividad industrial durante el periodo analizado.

\subsection{Sector terciario: concentración territorial de un sector en crecimiento}

$\mathrm{Al}$ analizar el sector terciario, se ratifica la tendencia concentradora del crecimiento económico al interior de la región Centro (cuadro 4), ya que en sólo nueve municipios de 553 se produce $66 \%$ del PIB terciario de la región, en tanto que contienen $21 \%$ de la población. El conjunto de esos municipios produjeron 299,000 millones de pesos en 2003, lo que representó un aumento de 31,000 millones de pesos en relación a 1999, con una aportación promedio de 3,400 millones de pesos en cada uno. Asi- 


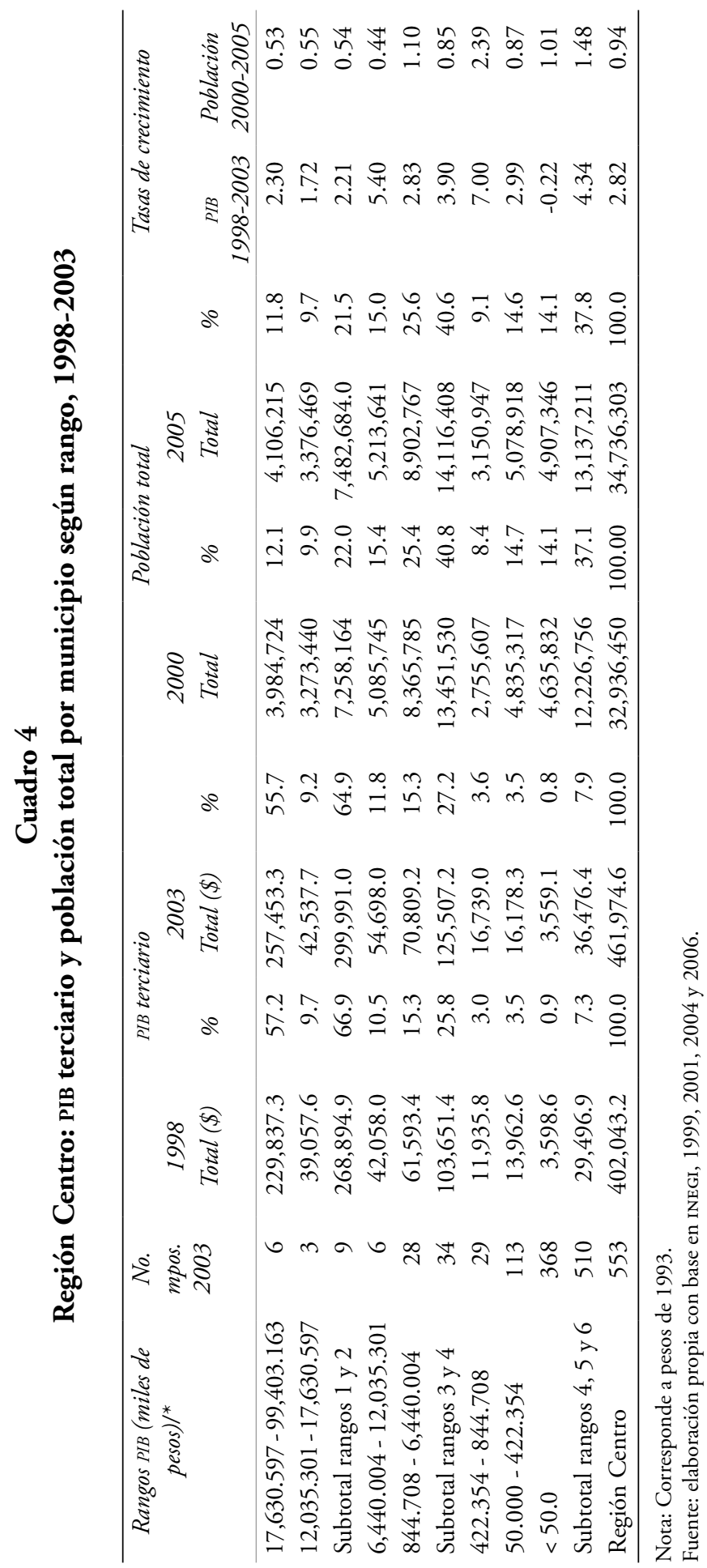


mismo, esos municipios -correspondientes a los rangos uno y dos según su aportación al PIB terciario- tienen una población de 7.4 millones de habitantes en 2005, de los cuales 224,000 se incorporaron en el último quinquenio; cada uno de los nueve municipios tendría una población promedio de 831,000 habitantes. Los valores del PIB sugieren que el terciario, ahí presente, corresponde en gran parte a actividades de alto valor agregado que requieren niveles altos de preparación escolar. Estas cifras, por otra parte, corroboran la reconversión económica de la región Centro hacia el sector terciario. ${ }^{8}$

El PIB terciario generado por los municipios de los rangos tres y cuatro entre 1998 y 2003 creció a una tasa promedio anual de $3.9 \%$, superior al promedio regional (2.8\%). En cada uno de los 34 municipios ubicados en esos rangos se habrían generado en promedio 642 millones de pesos. En ellos se genera el $27 \%$ del pIB total regional y habita $40 \%$ de la población; a cada municipio le corresponderían en promedio 415,000 habitantes.

Así, la mayor parte del aumento total del PIB regional en el quinquenio ocurrió en los cuatro primeros rangos de municipios con $88.5 \%$, que se reflejó también en un aumento relativo de la población de $49.5 \%$ o 889,000 personas.

En el extremo contrario, los rangos (cinco, seis y siete) más bajos del PIB terciario, corresponden a un total de 510 municipios, los cuales, no obstante haber tenido la TCPA más alta (4.3\%), su participación en el PIB total regional sólo aumentó de 7.3 a 7.9\% entre 1998 y 2003 con un aumento de casi 7,000 millones y un promedio de 13.6 millones en cada municipio. En estos rangos ocurrió el mayor aumento de población entre 2000 y 2005 con 910,000 habitantes o 50\% del aumento total en la región, y un promedio de 1,785 personas en cada municipio. En los rangos uno y dos el incremento promedio de población por municipio fue de 24,946 para un total de 224,520 , y en los rangos tres y cuatro el aumento por municipio fue de 19,555 para un total de 664,878.

El mayor aumento de la población en los tres últimos rangos en comparación con los municipios de los rangos uno y dos o tres y cuatro tiene que ver con que se trata, en primer lugar, de 510 municipios o $92.2 \% ;^{10}$

${ }^{8} \mathrm{Si}$ se considera solamente el rango uno al que pertenecen seis municipios, la concentración del sector es aún más evidente, dado que en ellos se generó 56\% del PIB terciario en 1993.

${ }^{9}$ Estos datos son demostrativos de la mayor importancia del sector terciario en la economía de la región Centro, toda vez que son tres veces superiores al PIB generado por el sector manufacturero en sus tres primeros rangos en los mismos años. Si se compara el PIB total terciario y el PIB total manufacturero, el primero es también tres veces superior en 1998 y 3.4 veces mayor en 2003 . Es por eso que el límite de los rangos es diferente en cada sector.

${ }^{10} \mathrm{La}$ población promedio de estos municipios es de 25,000 habitantes, por 831,000 de los municipios de los rangos uno y dos. 
en segundo lugar que se trata de localidades de entre 25,000 y 50,000 habitantes donde los servicios que ahí se desarrollan están principalmente orientados al consumidor y requieren de una población de menor calificación para su desarrollo; en tercer término, tiene que ver con el hecho de que los municipios de los rangos uno y dos son metropolitanos con tasas de crecimiento demográfico muy bajas e incluso negativas en algunos casos. De cualquier manera el dinamismo económico y demográfico de esos 510 municipios está influenciado por su relativa cercanía con las grandes ciudades en un radio no mayor a 100 kilómetros.

De lo seńalado hasta aquí es claro que durante el quinquenio 19982003, al mismo tiempo que continuó la tendencia a la desindustrialización relativa de la región Centro y la ZMCM, se fortaleció también su proceso de terciarización, cuyas actividades mantuvieron un crecimiento económico que, aunque moderado, destacaron en el ambiente recesivo que tuvo la economía nacional en su conjunto. Por otra parte, y aunque los municipios de los cuatro primeros rangos redujeron su participación en el PIB terciario regional en seis décimas porcentuales a favor de los municipios de los rangos cuatro, cinco y seis, su distribución es mayoritariamente metropolitana.

\subsection{Expresión territorial}

En el cuadro 5 que contiene las 14 unidades político-administrativas de mayor concentración del PIB terciario en la región, sobresale el hecho de que, en su totalidad, pertenecen a alguna zona metropolitana, siendo la ZMCM la que mayor peso tiene en el conjunto; los otros estados representados con algún municipio son Puebla y Querétaro solamente. No obstante, destaca que las TCPA de la población en el lapso 2000-2005 de los municipios ubicados en el primer y segundo contorno de crecimiento de la ZMCM son menores a uno por ciento o incluso negativas en cinco de ellos, en cada año. En una situación contraria se encuentran Toluca y Querétaro con tasas de crecimiento de 2.04 y $2.41 \%$, respectivamente, así como Puebla con $1.75 \%$. Estos espacios corresponderían probablemente a los únicos municipios metropolitanos centrales que atraen población de la periferia de sus propias áreas conurbadas y de las de las otras metrópolis regionales.

Como detalle peculiar, la mayoría de los municipios incluidos en esta lista son también los que concentran la actividad manufacturera de la región Centro, lo que corrobora la naturaleza metropolitana del crecimiento económico en la región, así como de su proceso de terciarización, en cierta medida vinculado con la actividad manufacturera por la demanda de servicios especializados que ésta tiene, pero también debido a que 


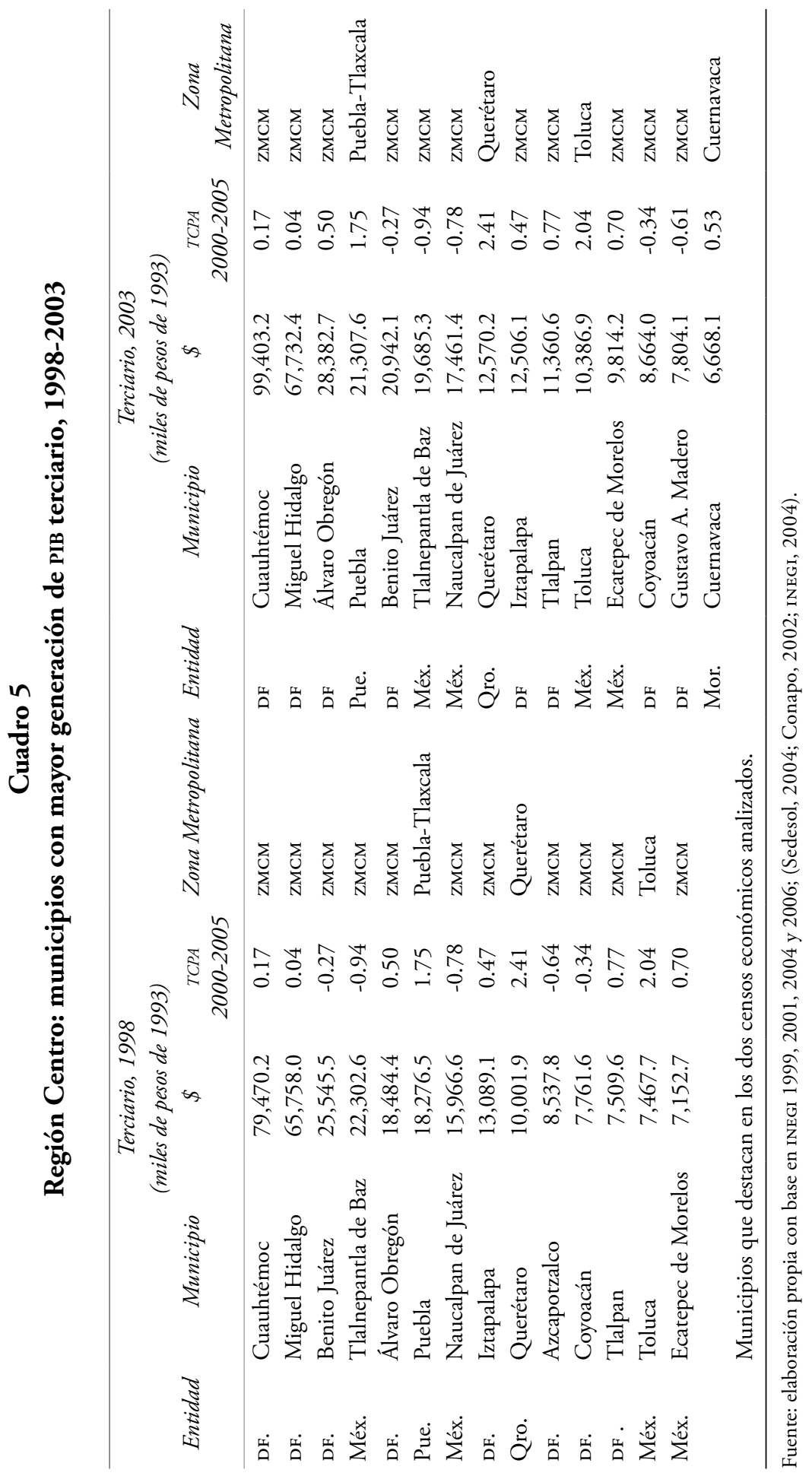


la ZMCM es la ciudad de mayor jerarquía en el Sistema Urbano Nacional, la cual concentra los servicios de más alta especialización y que mayor valor agregado generan.

Los mapas v y vi confirman lo antes descrito. La mayor proporción del PIB terciario se concentra en las zonas metropolitanas, y dentro de éstas, en los municipios centrales. Fuera de ellas solamente destacan San Juan del Río en Querétaro y Tehuacán en Puebla. Dicho en otros términos, el sector terciario de mayor especialización prevalece en los núcleos de población de alta jerarquía (más poblados) y en menor grado en ciudades medias y pequeñas; para este último caso, la hipótesis a contrastar en una investigación posterior, es que la expansión territorial del sector terciario en la región se debe a la difusión de servicios y comercios de baja especialización, que se distribuye conforme a la distribución de la población en general.

Las diferencias que más llaman la atención entre un mapa y otro es que, de 1998 a 2003, se incrementa la concentración del PIB terciario en las zonas metropolitanas; de hecho la formación del pIB en el sector servicios fuera de las zonas metropolitanas es poco significativa. Adicionalmente se aprecia que para 2003, los espacios vacios entre zonas metropolitanas los ocupan los municipios que registran una muy baja concentración del PIB terciario, lo que es posible interpretar como una tenue activación de economías locales (municipales) con base en el sector terciario de baja especialización.

Otro elemento que no se puede pasar por alto es que para 2003, la mayoría de municipios que cuentan con al menos una localidad de 5,000 habitantes o más, registran participación en el PIB terciario de la región. Por entidad, $82.7 \%$ del PIB terciario se concentra en el Distrito Federal y el Estado de México, y 10.6\% en Puebla y Querétaro, siendo el Estado de México y Querétaro los que tuvieron más altas tasas de crecimiento demográfico en el decenio 1995-2005.

Por otra parte, con respecto al siguiente punto de esta investigación, se realizará un análisis territorial de los procesos de migración interna reciente, con base en los resultados del II Conteo de Población y Vivienda 2005, para posteriormente, establecer las relaciones dinámicas entre grandes sectores económicos y migración interna. 


\section{Mapa $V$ \\ Región Centro: distribución territorial del PIB terciario, 1998}

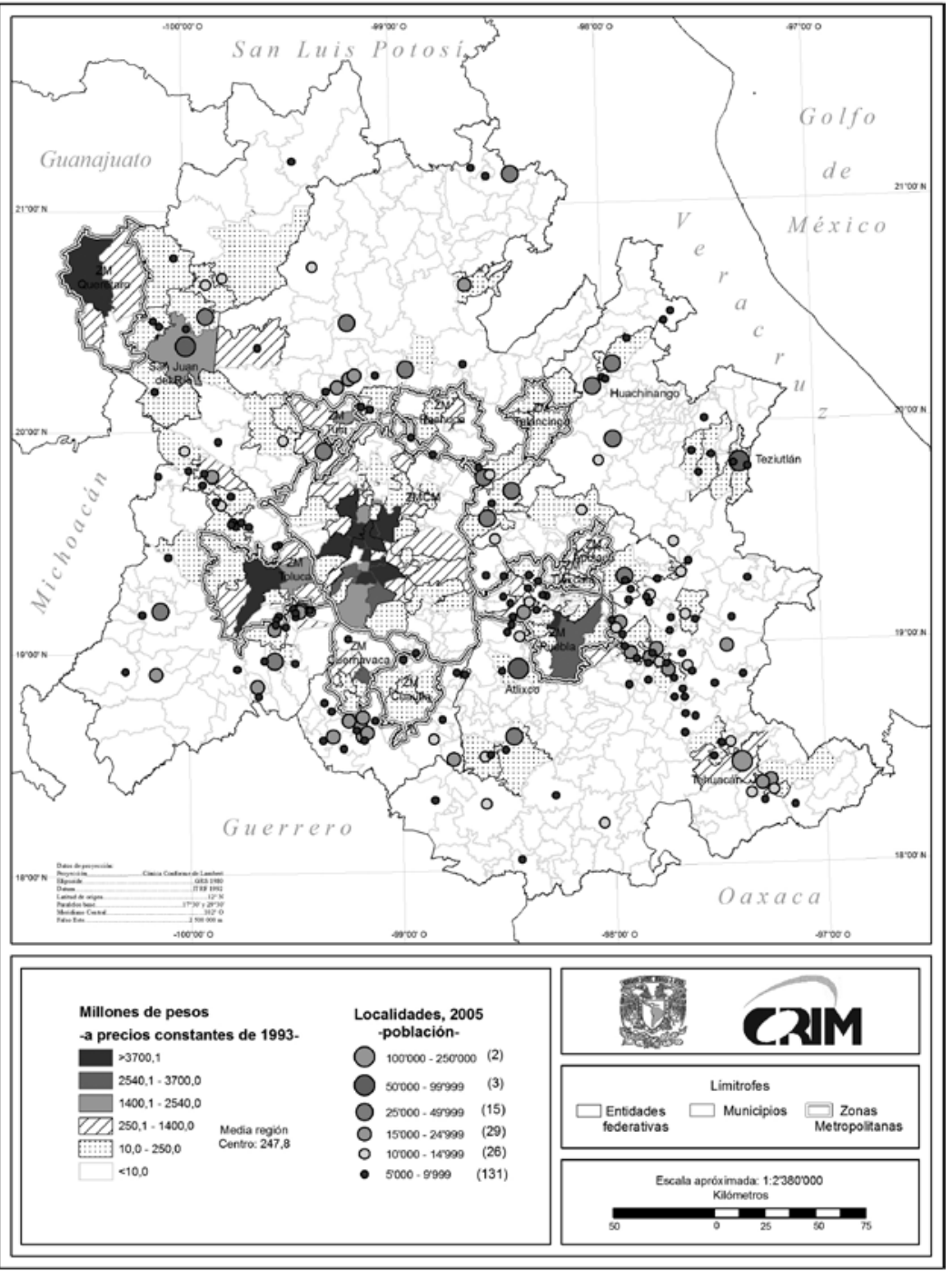

Fuente: Elaboración propia con base en INEGi 1999, 2000; (Sedesol, 2004; Conapo, 2002; INEGI, 2004). 


\section{Mapa VI}

\section{Región Centro: distribución territorial del PIB terciario, 2003}

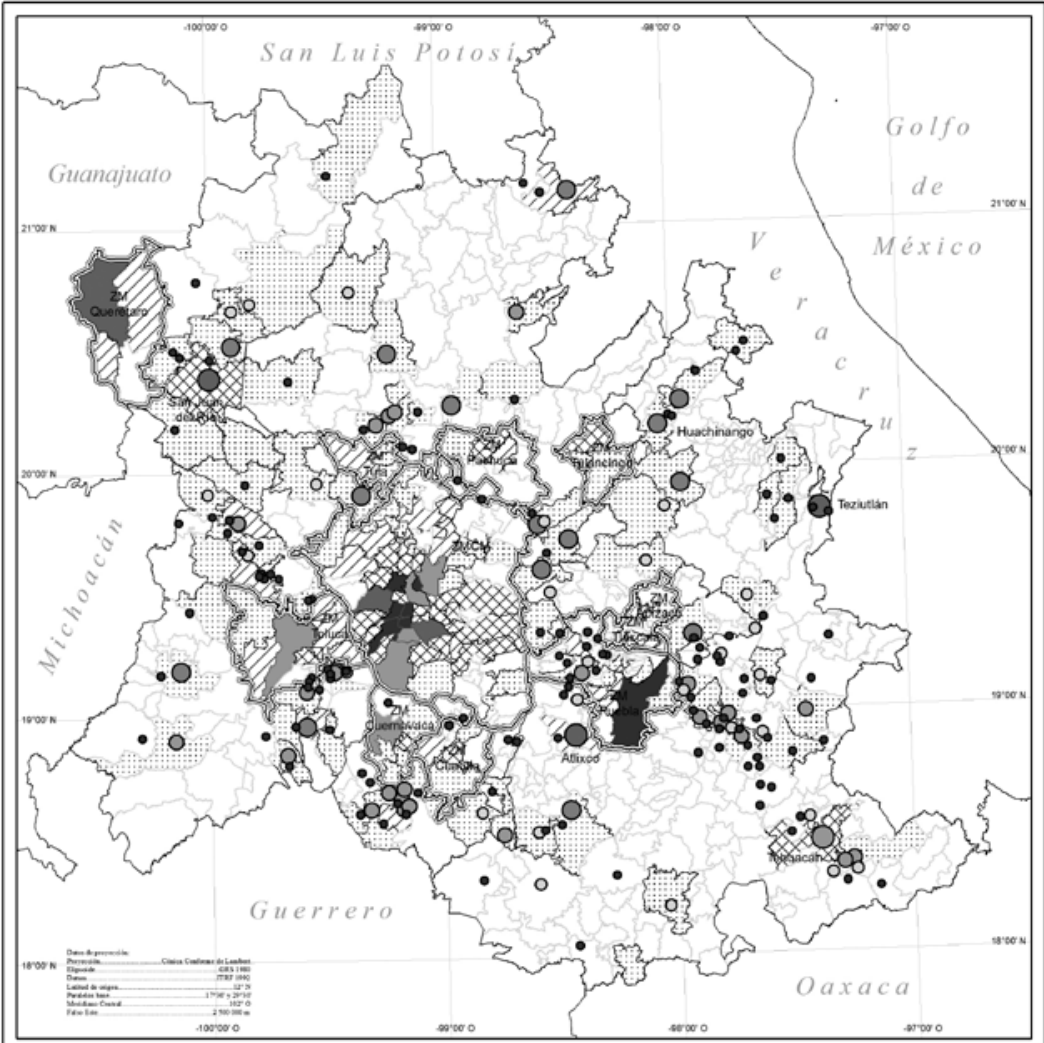

Millones de pesos

-a precios constantes de 1993 .

$>17500,1$

Localidades, 2005 -población-

$12000,1 \cdot 17500,0$

$6450,1 \cdot 120000$

28080,1 - 6450,0

V7] $425,1-850.0$

i.i. $50.0-425.0$

$<50,0$

$100000-250000$ (2)

$50000 \cdot 99999 \quad$ (3)

$25000-49999$ (15)

O $15000 \cdot 24999 \quad(29)$

O $10000-14999$ (26)

- $5000-9999$ (131)

(1)

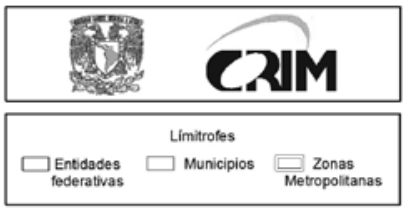

Escala apróximada: 1:2380000

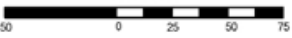

Fuente: Elaboración propia con base en INEGi 2004, 2005; (Sedesol, 2004; Conapo, 2002; INEGI, 2004). 


\section{La migración interna reciente}

En esta sección se revisan algunas características generales de la migración reciente ${ }^{11}$ en la región Centro, así como de los migrantes, en particular en lo que se refiere a género y edad.

En primer término, es notorio que es de las mismas entidades que conforman la región Centro de donde proviene la mayor parte de inmigrantes recientes hacia ella, al representar $68.5 \%$ del total, lo que es un claro indicio de la fuerte dinámica migratoria intrarregional. Encabezan la lista el Distrito Federal (38.2\%), el Estado de México (17.3\%) y Puebla (5.5\%) (cuadro 6).

De las entidades no pertenecientes a la región Centro, las que mayor número de inmigrantes aportan son Veracruz (7.3\%) en primer lugar; Oaxaca (3.9\%) y Guerrero (3.5\%) en segundo lugar, Michoacán (2.3\%), Guanajuato (2.2\%), Jalisco (1.9\%) y Chiapas (1.8\%) en tercero, y por último las entidades restantes.

Por género, destaca el hecho que a escala tanto inter como intraregional, son las mujeres quienes arriban en mayor número a la región $(52 \%$ en promedio). De acuerdo a las características de los nuevos procesos de reestructuración territorial, así como al dinamismo de los nuevos espacios económicos en el país y concretamente en la región Centro, reconocer la mano de obra por género es importante, debido a: 1) la proliferación de industrias o tipos de servicios que demandan abundante mano de obra femenina, lo que promueve la formación de mercados laborales feminizados primordialmente de baja calificación, vinculados con empresas medianas y pequeñas; 2) el impacto de la cantidad de mano de obra en los niveles de productividad, debido a la presencia de actividades que hacen uso intensivo de la fuerza de trabajo (De la Garza, 1994: 54-55, 59), y 3 ) el mayor acceso que tienen las mujeres para insertarse laboralmente en el sector terciario, sobre todo en actividades informales (Corona y Luque, 1992; Morelos, 2005).

De igual forma, el aumento de la incorporación laboral de las mujeres es consecuencia de las etapas de crisis que han afectado al país desde el decenio de 1980, lo que obliga a las mujeres a incorporarse al sector formal o informal (o el subempleo) para ayudar a la economía familiar (Escamilla, 2003: 258).

En suma, la inmigración a la región Centro se origina en todas las entidades de la república en mayor o menor volumen, si bien a mayor distancia disminuye el número de inmigrantes, asimismo, después de las

\footnotetext{
${ }^{11}$ Como migración reciente se considera a la población que, en el lapso de los cinco años previos al levantamiento del censo de población, cambió su localidad de residencia.
} 


\section{Cuadro 6 \\ México: emigración desde las entidades federativas hacia la región Centro, 2005}

\begin{tabular}{|c|c|c|c|c|c|c|}
\hline \multirow[t]{2}{*}{$\begin{array}{c}\text { Entidad } \\
\text { Federativa }\end{array}$} & \multicolumn{5}{|c|}{ Emigrantes } & \multirow{2}{*}{$\begin{array}{l}\text { Participación (\%) } \\
\text { por entidad } \\
\text { federativa de la } \\
\text { emigración hacia } \\
\text { la región Centro }\end{array}$} \\
\hline & Total & Hombres & Mujeres & $\begin{array}{c}\text { Hombres } \\
(\%)\end{array}$ & $\begin{array}{l}\text { Mujeres } \\
(\%)\end{array}$ & \\
\hline Aguascalientes & 3,772 & 1,850 & 1,922 & 49.0 & 51.0 & 0.4 \\
\hline Baja California & 9,629 & 4,946 & 4,683 & 51.4 & 48.6 & 1.0 \\
\hline Baja California Sur & 1,276 & 666 & 610 & 52.2 & 47.8 & 0.1 \\
\hline Campeche & 1,788 & 932 & 856 & 52.1 & 47.9 & 0.2 \\
\hline Coahuila & 4,365 & 2,225 & 2,140 & 51.0 & 49.0 & 0.5 \\
\hline Colima & 1,589 & 818 & 771 & 51.5 & 48.5 & 0.2 \\
\hline Chiapas & 16,729 & 8,347 & 8,382 & 49.9 & 50.1 & 1.8 \\
\hline Chihuahua & 6,101 & 3,135 & 2,966 & 51.4 & 48.6 & 0.7 \\
\hline Distrito Federal* & 360,642 & 177,718 & 182,924 & 49.3 & 50.7 & 38.8 \\
\hline Durango & 2,202 & 1,101 & 1,101 & 50.0 & 50.0 & 0.2 \\
\hline Guanajuato & 20,321 & 9,204 & 11,117 & 45.3 & 54.7 & 2.2 \\
\hline Guerrero & 31,881 & 14,389 & 17,492 & 45.1 & 54.9 & 3.4 \\
\hline Hidalgo* & 26,819 & 12,308 & 14,511 & 45.9 & 54.1 & 2.9 \\
\hline Jalisco & 17,469 & 8,518 & 8,951 & 48.8 & 51.2 & 1.9 \\
\hline México* & 160,283 & 76,686 & 83,597 & 47.8 & 52.2 & 17.3 \\
\hline Michoacán & 21,192 & 9,358 & 11,834 & 44.2 & 55.8 & 2.3 \\
\hline Morelos* & 15,418 & 7,300 & 8,118 & 47.3 & 52.7 & 1.7 \\
\hline Nayarit & 1,436 & 703 & 733 & 49.0 & 51.0 & 0.2 \\
\hline Nuevo León & 9,012 & 4,691 & 4,321 & 52.1 & 47.9 & 1.0 \\
\hline Oaxaca & 36,190 & 16,493 & 19,697 & 45.6 & 54.4 & 3.9 \\
\hline Puebla* & 50,879 & 23,180 & 27,699 & 45.6 & 54.4 & 5.5 \\
\hline Querétaro* & 9,996 & 4,665 & 5,331 & 46.7 & 53.3 & 1.1 \\
\hline Quintana Roo & 5,396 & 2,734 & 2,662 & 50.7 & 49.3 & 0.6 \\
\hline San Luis Potosí & 6,585 & 3,120 & 3,465 & 47.4 & 52.6 & 0.7 \\
\hline Sinaloa & 5,106 & 2,514 & 2,592 & 49.2 & 50.8 & 0.5 \\
\hline Sonora & 3,812 & 1,920 & 1,892 & 50.4 & 49.6 & 0.4 \\
\hline Tabasco & 6,536 & 3,248 & 3,288 & 49.7 & 50.3 & 0.7 \\
\hline Tamaulipas & 7,215 & 3,722 & 3,493 & 51.6 & 48.4 & 0.8 \\
\hline Tlaxcala* & 12,134 & 5,891 & 6,243 & 48.5 & 51.5 & 1.3 \\
\hline Veracruz & 67,884 & 31,584 & 36,300 & 46.5 & 53.5 & 7.3 \\
\hline Yucatán & 2,950 & 1,427 & 1,523 & 48.4 & 51.6 & 0.3 \\
\hline Zacatecas & 2,403 & 1,102 & 1,301 & 45.9 & 54.1 & 0.3 \\
\hline
\end{tabular}


Continúa Cuadro 6...

\begin{tabular}{|c|c|c|c|c|c|c|}
\hline \multirow[t]{2}{*}{$\begin{array}{c}\text { Entidad } \\
\text { Federativa }\end{array}$} & & hacia la $n$ & $\begin{array}{l}\text { Emigrantes } \\
\text { egión Cent }\end{array}$ & tro, 2005 & & \multirow{2}{*}{$\begin{array}{c}\text { Participación (\%) } \\
\text { por entidad } \\
\text { federativa de la } \\
\text { emigración hacia } \\
\text { la región Centro }\end{array}$} \\
\hline & Total & Hombres & Mujeres & $\begin{array}{c}\text { Hombres } \\
(\%)\end{array}$ & $\begin{array}{c}\text { Mujeres } \\
(\%)\end{array}$ & \\
\hline $\begin{array}{l}\text { Entidades NO } \\
\text { incluidas en la } \\
\text { región Centro }\end{array}$ & 292,839 & 138,747 & 154,092 & 47.4 & 52.6 & 31.5 \\
\hline $\begin{array}{l}\text { Entidades } \\
\text { SI incluidas } \\
\text { en la región Centro }\end{array}$ & 636,171 & 307,748 & 328,423 & 48.4 & 51.6 & 68.5 \\
\hline $\begin{array}{l}\text { Total nacional de } \\
\text { inmigrantes a la } \\
\text { región Centro }\end{array}$ & 929,010 & 446,495 & 482,515 & 48.1 & 51.9 & 100.0 \\
\hline
\end{tabular}

*Entidad que forma parte de la región Centro.

Fuente: elaboración propia con base en INEGI, 2006.

propias entidades de la región Centro, es desde las entidades de mayor atraso económico (Oaxaca y Guerrero por ejemplo) de donde la región Centro recibe mayor número de migrantes. Sumado a ello el hecho de que los inmigrantes son mayoritariamente población femenina, es posible plantear otra hipótesis adicional a la original: a los municipios de mayor dinamismo económico de la región Centro, llega mano de obra de baja especialización en busca de emplearse de manera formal (o informal) en el sector económico secundario o terciario, principalmente. ${ }^{12}$ Estos municipios receptores serían los que en este ejercicio se identificaron como los que mayor PIB reportaron en los diferentes sectores de actividad. La migración intrarregional, sin embargo, también tendría la misma dirección en sus flujos.

Pero por otra parte, si bien no es posible asociar de forma determinista la dinámica económica sectorial de los municipios y las entidades de la región Centro a su dinámica migratoria y demográfica, sí es posible hacer algunas observaciones generales en el sentido de que las mayores concentraciones poblacionales, económicas y urbanas, de carácter metropolitano, son las principales atractoras de los flujos de migración reciente nacional y sobre todo intrarregional. Estas metrópolis se localizan en las entidades más pobladas y concentran las actividades manufactureras y de servicios, como ya se analizó.

Como un elemento para comprobar la hipótesis planteada, el siguiente paso es analizar la dinámica migratoria de los municipios de las principales metrópolis de la región, y comprobar si existe relación entre los

\footnotetext{
${ }^{12}$ Esta hipótesis queda agendada para desarrollarse en una próxima investigación.
} 
flujos migratorios y las más importantes concentraciones del PIB en los diferentes sectores. Los datos siguientes sustentan esta idea.

En primer lugar, de los poco más de 2.4 millones de migrantes internos nacionales: a) 1.6 millones $(68.7 \%)$ arribaron a municipios pertenecientes a zonas metropolitanas; $y$ b) casi 0.93 millones (38.6\%) tuvieron como destino algún municipio de la región Centro (cuadro 7). El Estado de México sobresale por ser la entidad que absorbió casi la mitad de los inmigrantes llegados a la región con 44.9\%; seguido por el Distrito Federal (20.2\%), Puebla (10.4\%) y Querétaro (7.4\%). En conjunto, el Estado de México y el Distrito Federal absorbieron 65\% de los inmigrantes de la región.

\section{Cuadro 7 \\ Región Centro: total de inmigrantes por entidad federativa, 2005}

\begin{tabular}{lrrrrrr}
\hline Entidad Federativa & \multicolumn{5}{c}{ Inmigrantes } \\
& \multicolumn{1}{c}{ Totales } & & \multicolumn{3}{c}{ Porcentajes } \\
& Totales & Hombres & Mujeres & Totales & Hombres & Mujeres \\
\hline Distrito Federal & 187,363 & 86,899 & 100,464 & 20.2 & 19.5 & 20.8 \\
Hidalgo & 74,242 & 35,902 & 38,340 & 8.0 & 8.0 & 7.9 \\
México & 416,778 & 202,202 & 214,576 & 44.9 & 45.3 & 44.5 \\
Morelos & 57,021 & 27,660 & 29,361 & 6.1 & 6.2 & 6.1 \\
Puebla & 96,503 & 46,885 & 49,618 & 10.4 & 10.5 & 10.3 \\
Querétaro & 69,140 & 33,524 & 35,616 & 7.4 & 7.5 & 7.4 \\
Tlaxcala & 27,963 & 13,423 & 14,540 & 3.0 & 3.0 & 3.0 \\
Región Centro & 929,010 & 446,495 & 482,515 & 100.0 & 100.0 & 100.0 \\
\hline
\end{tabular}

Fuente: elaboración propia con base en INEGI, 2006.

Mayor detalle sobre el destino de los inmigrantes se muestra en el cuadro 8, en donde se indica que de los casi 0.93 millones de personas que llegaron a la región Centro, 0.8 millones lo hicieron a los municipios pertenecientes a alguna zona metropolitana, lo que permite destacar el carácter inminentemente metropolitano de los flujos migratorios a la región Centro. La zMCM, fue el destino de 70\% de los inmigrantes metropolitanos que arribaron a la región Centro; en segundo lugar, pero bastante lejos de este núcleo central, se ubican las zonas metropolitanas de Querétaro y Puebla-Tlaxcala, y en tercer lugar, las zonas metropolitanas de Cuernavaca, Pachuca y Toluca.

A raíz de lo anterior se pueden expresar las primeras conclusiones parciales: la ZMCM se mantiene como la principal receptora de los flujos migratorios en la región, en tanto que las zonas metropolitanas más próximas a ésta (en un radio de entre 100 y 150 kilómetros), se colocan en segundo como receptoras de los flujos migratorios intra e interregionales. 


\section{Cuadro 8}

Región Centro: total de inmigrantes por zona metropolitana, 2005

\begin{tabular}{lrrrrrr}
\hline \multicolumn{1}{c}{ Zona } & \multicolumn{5}{c}{ Inmigrantes } \\
\multicolumn{1}{c}{ Metropolitana } & \multicolumn{2}{c}{ Totales } & & \multicolumn{3}{c}{ Porcentajes } \\
& Totales & Hombres & Mujeres & Totales & Hombres & Mujeres \\
\hline ZM Apizaco & 6,911 & 3,345 & 3,566 & 0.9 & 0.9 & 0.8 \\
zM Ciudad de México & 570,000 & 272,346 & 297,654 & 70.5 & 70.2 & 70.8 \\
zM Cuautla & 13,446 & 6,717 & 6,729 & 1.7 & 1.7 & 1.6 \\
zM Cuernavaca & 35,451 & 17,020 & 18,431 & 4.4 & 4.4 & 4.4 \\
zM Pachuca & 26,527 & 12,878 & 13,649 & 3.3 & 3.3 & 3.2 \\
zM Puebla-Tlaxcala & 52,744 & 25,480 & 27,264 & 6.5 & 6.6 & 6.5 \\
zM Querétaro & 53,177 & 25,667 & 27,510 & 6.6 & 6.6 & 6.5 \\
zM San Martín & 2,065 & 969 & 1,096 & 0.3 & 0.2 & 0.3 \\
Texmelucan & & & & & & \\
zM Tlaxcala & 7,829 & 3,795 & 4,034 & 1.0 & 1.0 & 1.0 \\
ZM Toluca & 28,345 & 14,038 & 14,307 & 3.5 & 3.6 & 3.4 \\
zM Tula & 6,260 & 3,038 & 3,222 & 0.8 & 0.8 & 0.8 \\
zM Tulancingo & 6,063 & 2,909 & 3,154 & 0.7 & 0.7 & 0.7 \\
Total & 808,818 & 388,202 & 420,616 & 100.0 & 100.0 & 100.0 \\
\hline
\end{tabular}

Fuente: elaboración propia con base en INEGI, 2006.

Por otra parte, es preciso referir que en tanto el promedio nacional de inmigrantes por municipio es de 981; para la región Centro es de 1,680. Asimismo, el promedio de inmigrantes por zona metropolitana en la región Centro es de 30,055 si se incluye la ZMCM, y de 20,056 si se excluye.

Ahora bien, considerando que una de las hipótesis de esta investigación es que los flujos de inmigrantes en el país en general y en la región Centro en particular, tienen una connotación eminentemente laboral, un elemento que abona en este sentido es el que aporta la identificación de la edad de los migrantes que llegan a la región, concretamente a los municipios de mayor dinamismo económico que forman parte de las zonas metropolitanas.

Según se observa en la gráfica I, casi $50 \%$ de los inmigrantes que llegan a alguna entidad de la región Centro está en el grupo de edad de 15 a 34 años, lo que los ubica en el sector de Población Económicamente Activa (PEA) que demanda un empleo. Pero si se amplia el rango de edad de los 15-49 años ${ }^{13}$ el porcentaje se incrementa a 70\%, y aún más si se considera a la población infantil (grupo entre 5-14 ańos) como pola PEA.

${ }^{13}$ Para esta investigación, la edad de 50 años se considera como el umbral para pertenecer a 


\section{Gráfica I}

\section{Región Centro: total de inmigrantes por entidad federativa, 2005}

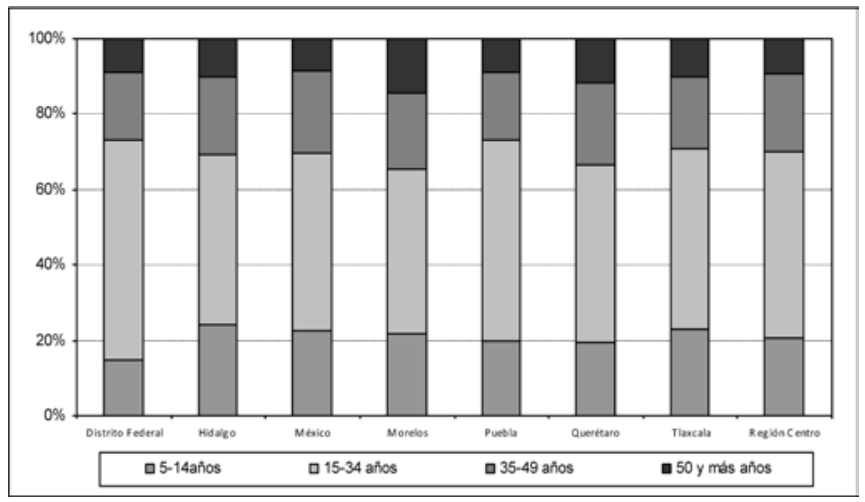

Fuente: elaboración propia con base en INEGI, 2006.

\section{Gráfica II}

\section{Región Centro: total de inmigrantes por zona metropolitana, 2005}

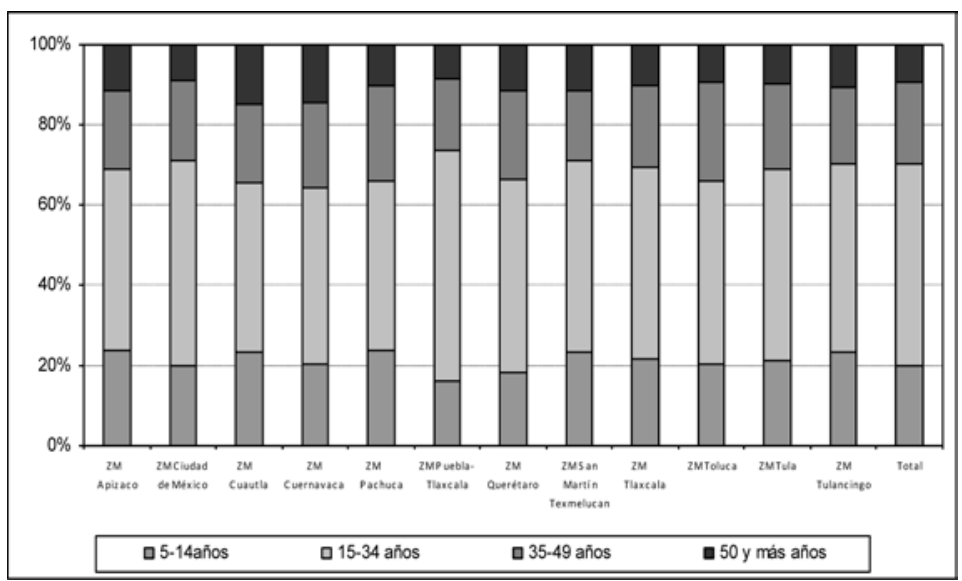

Fuente: elaboración propia con base en INEGI, 2006.

tencial a incluirse a la $\mathrm{PEA}^{14}$ el porcentaje queda en $90.5 \%$; esto probablemente indicaría una migración familiar. El grupo de 50 y más años tiene un peso de $9.6 \%$ del promedio regional, lo que permitiría suponer, también, un escenario de migración de retorno (Negrete, 1999:342), con miras al retiro.

${ }^{14}$ Ante los efectos de la recesión y crisis económica en México, cada vez un mayor número de miembros de las familias se incorporan al trabajo informal -mujeres y menores de edad- (Escamilla, 2003: 262). 


\section{Mapa VII \\ Región Centro: migración interna municipal, 2005}

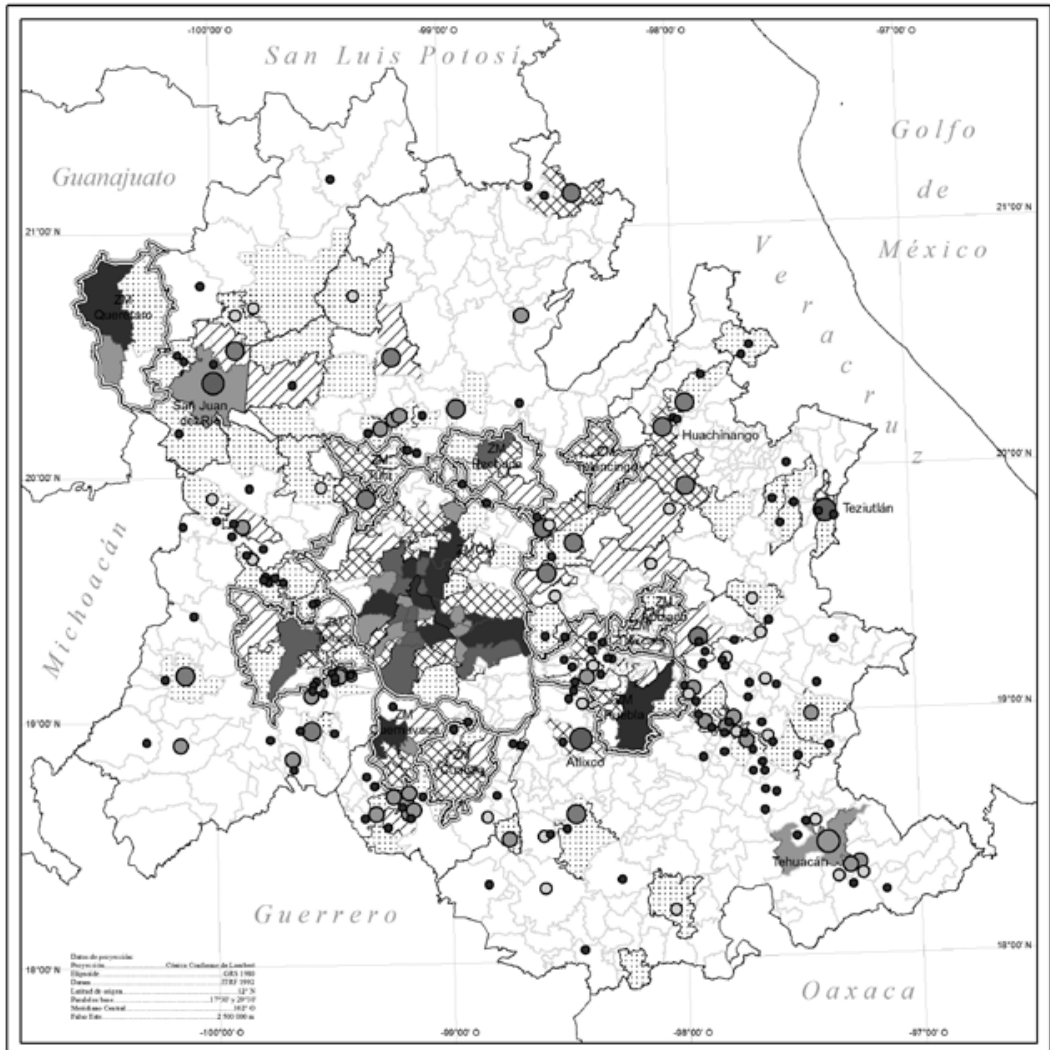

Total de inmigrantes provenientes de

cualquier entidad federativa, 2005

Localidades, 2005 -población-

$>17501$

$100000 \cdot 250000$ (2)

$12501 \cdot 17500$

$50000-92099$

7001 - 12500

$\triangle 21501-7000$

DD 1001 - 1500

$\begin{array}{lll}\bigcirc & 25000-49999 & (15) \\ & 15000-24999 & (29)\end{array}$

E: $501-1000$

Media region
Centro: 1680

O $10000-14999$ (26)

$<500$

- $5000-9909$ (131)

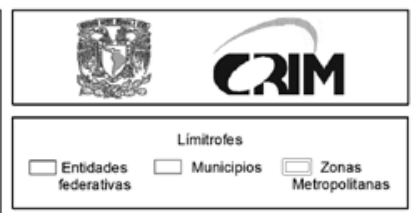

Escala aprónimada: 1:2380000

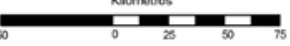

Fuente: Elaboración propia con base en INEGI (2005); (Sedesol, 2004; Conapo, 2002; INEGI, 2004). 
Para el caso específico de las zonas metropolitanas, los porcentajes no presentan mucha variación respecto al registrado en la región. En la gráfica II se identifica que el patrón migratorio por edades hacia las zonas metropolitanas tiene el mismo carácter inminentemente laboral que la región Centro, pero para encontrar las diferencias porcentuales y de escala de análisis, es necesario trasladar los resultados a su representación territorial.

En el mapa VII se observa que son los municipios metropolitanos y específicamente los municipios centrales de las zonas metropolitanas, los territorios receptores del mayor número de inmigrantes en la región. Este resultado establece los primeros nexos para ratificar la hipótesis sobre el carácter inminentemente laboral que tiene la migración interna en el país y particularmente en la región Centro.

En segundo lugar, fuera de las zonas metropolitanas, únicamente sobresalen los municipios de San Juan del Río en Querétaro, Tepeji del Río en el estado de Hidalgo, y Atlixco, Tehuacán y Teziutlán en Puebla. El rasgo característico de estos municipios es que cuentan con localidades entre 50,000 y 250,000 habitantes, lo que permite suponer la presencia de mercados de trabajo subregionales, pero por su ubicación próxima a las zonas metropolitanas o en puntos de intersección de los ejes carreteros más importantes de la región, es posible considerarlos supeditados a la influencia económica de las zonas metropolitanas cercanas.

En tercer lugar, los rangos más bajos de inmigración tienen una ubicación radioconcéntrica alrededor de las zonas metropolitanas, en general, y de la ZMCM en particular, lo que permite inferir que existe una dependencia económico-funcional (commuting) de estos municipios de baja inmigración con los municipios metropolitanos de mayor dinamismo económico (Corona y Luque, 1992).

Otra observación interesante es la ubicación de los municipios de baja inmigración a lo largo de los ejes carreteros que cruzan la región. Destacan dos casos: el primero es el corredor que se forma desde el noreste de la ZMCM para enlazar la ZM de Tulancingo y posteriormente conectarse (fuera de la región Centro), con la zM de Poza Rica, y el segundo caso es el corredor que enlaza a la zмcM con la zм de Puebla y las zonas metropolitanas de Xalapa, Córdoba y Orizaba que fungen como nodos de conectores y de acceso hacia la costa del Golfo de México.

Otra manera de analizar la inmigración a la región Centro es calculando una tasa de inmigración, que desde otra óptica, permita apreciar el peso relativo del total de inmigrantes en función de la población total por municipio. De acuerdo con el mapa viII, un primer aspecto a destacar es la complejización del patrón migratorio identificado, sustentado en la disminución del peso que tienen los inmigrantes al interior de las zonas 


\section{Mapa VIII}

\section{Región Centro: Tasa de inmigración interna municipal, 2005}

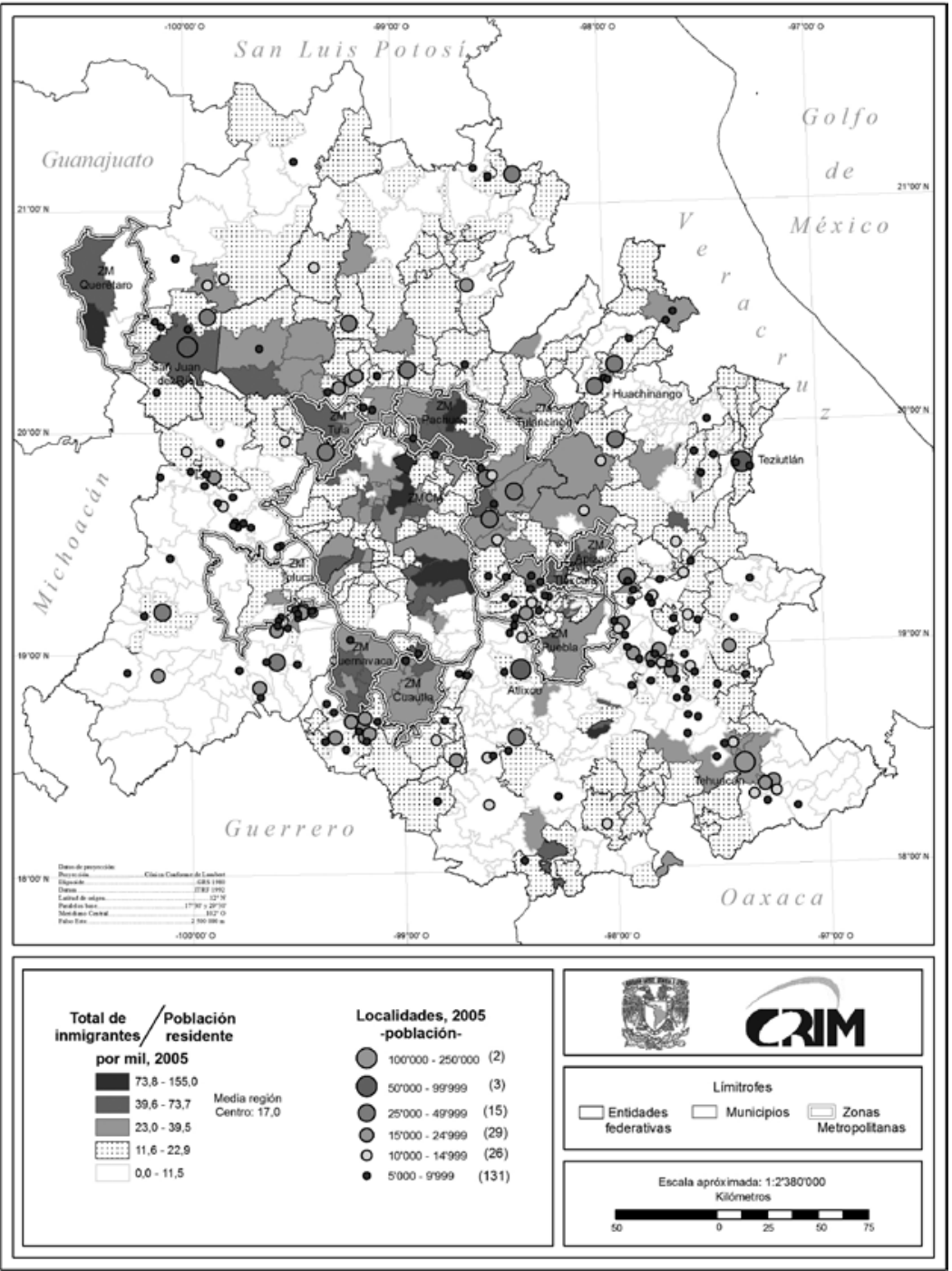

Fuente: Elaboración propia con base en INEGI (2005); (Sedesol, 2004; Conapo, 2002; INEGI, 2004). 
metropolitanas y concretamente de la zмсм, ya que dejan de ser los municipios centrales los de mayor peso inmigratorio, y son los municipios metropolitanos circundantes a los municipios centrales, los que destacan en el conjunto regional.

Este dato obliga a referir la hipótesis sobre la intensidad migratoria inter e intrametropolitana, que aluden a la salida de la Población Económicamente Activa (PEA) de alta calificación (y de mayor poder adquisitivo) a los municipios suburbanos de las zonas metropolitanas y el arribo de PEA de baja calificación a los municipios centrales de esas mismas zonas (Geyer, 1996).

Con este nivel de análisis, se realiza una depuración del estudio hasta aquí realizado, ya que se debe tener en consideración que, si bien, la inmigración en términos absolutos se registra en los municipios centrales de las zonas metropolitanas, en términos relativos la inmigración tiene mayor impacto en los municipios periféricos de las mismas zonas metropolitanas o de sus espacios periurbanos, lo que, por otra parte, no se contrapone con la hipótesis sobre el carácter laboral del proceso inmigratorio a la región de estudio.

\section{Conclusiones}

El análisis realizado en esta investigación sobre dinamismo económico por gran sector económico para los municipios de la región Centro, así como sus tendencias inmigratorias, en conjunto, permite esbozar las siguientes aseveraciones a manera de conclusión:

La dinámica económica en la región Centro, se especializa en el sector secundario, y particularmente en el terciario.

Como se identificó, el sector primario pierde importancia (absoluta y relativa) ante el crecimiento del secundario y principalmente el terciario, que se perfilan como los sectores que marcan la pauta de la dinámica económica en la región Centro.

Por otra parte, el secundario se reestructura territorialmente, proceso evidenciado por una dispersión territorial de las manufacturas hacia ciudades medias y pequeñas al interior de la región Centro, mientras que el terciario registra un proceso diferenciado: los servicios y el comercio especializado se localizan en zonas metropolitanas o núcleos urbanos de alta jerarquía, mientras que los servicios y el comercio de baja especialización, se distribuyen en localidades de baja jerarquía urbana.

Los espacios económicamente más dinámicos en la región Centro son los metropolitanos.

Si bien la dinámica económica al interior de la región Centro se enfoca en los sectores secundario y terciario, son las zonas metropolitanas y 
concretamente sus municipios centrales, los verdaderos territorios protagonistas de la dinámica económica, al concentrarse en ellos los porcentajes más altos del PIB secundario y terciario de la región. Esta tendencia será difícil de revertir debido a la especialización terciaria que acontece en el conjunto del país y concretamente en la región Centro.

La inmigración hacia la región Centro posee un carácter eminentemente laboral y un destino territorial preferentemente metropolitano.

Se constata la capacidad de atracción migratoria de las entidades federativas que integran la región Centro del país, a escala de sus municipios, en particular de los metropolitanos, pues son las zonas metropolitanas las que concentran $76.2 \%$ de la población de la región Centro, $22.9 \%$ del PIB primario; 93\% del PIB manufacturero, у 95.3\% del PIв terciario (cuadros A.2). Esto se observa en los mapas que representan la distribución de las actividades económicas a nivel municipal.

La tendencia actual del fenómeno migratorio en la región Centro está intimamente relacionada con el género y la calificación para el empleo de los inmigrantes.

Si bien fue validado el carácter eminentemente laboral del proceso migratorio al interior de la región Centro, se identificó que hay un fuerte sesgo en cuanto al género de los inmigrantes, debido a las tendencias que se marcan al interior de los procesos productivos, cuyo rasgo característico es optar por la contratación de mano de obra femenina y poco calificada, lo que reduce el costo de la producción e incrementa la tasa de ganancia (Morelos, 2005). Un ejemplo es la industria del vestido.

Temas pendientes dentro de una agenda de investigación entre economía y migración.

Para finalizar, entre los temas que se reconoce quedan pendientes dentro de una agenda de trabajo: 1) ahondar en el análisis por ramas y subsectores al interior de los tres grandes sectores de actividad económica para obtener mejores elementos de análisis y reconocer específicamente en donde se está dinamizando la economía de la región Centro; 2) analizar la calificación para el trabajo de los inmigrantes para evaluar su capacidad de inserción a los mercados de empleo y medir su impacto en la estructura y la dinámica económica a diferentes escalas (local, regional), y 3$)$ profundizar en la identificación de la edad y el género de los migrantes para evaluar la tendencia que siguen los mercados de trabajo y prever su impacto en las disparidades regionales. 


\begin{abstract}
Anexo
Cuadro A.1

Región Centro: concentración del PIB por zonas metropolitanas, 1998-2003
\end{abstract}

\begin{tabular}{|c|c|c|c|c|c|c|}
\hline \multirow{3}{*}{$\begin{array}{c}\text { Zona } \\
\text { Metropolitana } \\
\text { (miles de pesos de } \\
\text { 1993) }\end{array}$} & \multicolumn{6}{|c|}{ Producto Interno Bruto, 1998} \\
\hline & \multicolumn{3}{|c|}{ Total } & \multicolumn{3}{|c|}{$\%$} \\
\hline & Primario & Secundario & Terciario & Primario & Secundario & Terciario \\
\hline $\begin{array}{l}\text { zM Ciudad } \\
\text { de México }\end{array}$ & $4,319.0$ & $89,055.4$ & $319,821.3$ & 31.3 & 73.3 & 83.8 \\
\hline zм Puebla-Tlaxcala & 448.6 & $9,306.5$ & $20,614.6$ & 3.3 & 7.7 & 5.4 \\
\hline zM Toluca & 245.0 & $10,030.8$ & $10,014.1$ & 1.8 & 8.3 & 2.6 \\
\hline zM Querétaro & 2.8 & $5,098.8$ & $10,674.0$ & 0.0 & 4.2 & 2.8 \\
\hline zM Cuernavaca & $8,529.9$ & $3,401.4$ & $8,215.3$ & 61.8 & 2.8 & 2.2 \\
\hline zM Pachuca & 227.3 & 382.8 & $4,406.5$ & 1.6 & 0.3 & 1.2 \\
\hline zM Cuautla & 0.0 & 319.6 & $2,217.0$ & 0.0 & 0.3 & 0.6 \\
\hline zM Tlaxcala & 0.0 & 343.3 & $1,458.4$ & 0.0 & 0.3 & 0.4 \\
\hline zM Tulancingo & 10.4 & 287.0 & $1,442.5$ & 0.1 & 0.2 & 0.4 \\
\hline zM Tula & 4.4 & $1,902.7$ & $1,368.0$ & 0.0 & 1.6 & 0.4 \\
\hline zM Apizaco & 3.9 & 799.6 & $1,542.8$ & 0.0 & 0.7 & 0.4 \\
\hline $\begin{array}{l}\text { zM San Martín } \\
\text { Texmelucan }\end{array}$ & 0.2 & 631.3 & 668.6 & 0.0 & 0.5 & 0.2 \\
\hline Total metropolitano & $13,791.5$ & $121,559.1$ & $382,443.1$ & 100.0 & 100.0 & 100.0 \\
\hline Total región Centro & $19,476.9$ & $131,075.3$ & $402,043.2$ & & & \\
\hline $\begin{array}{l}\text { Porcentaje } \\
\text { metropolitano } \\
\text { de la región Centro }\end{array}$ & 70.8 & 92.7 & 95.1 & & & \\
\hline Zona & \multicolumn{6}{|c|}{ Producto Interno Bruto, 2003} \\
\hline Metropolitana & \multicolumn{3}{|c|}{ Total } & \multicolumn{3}{|c|}{$\%$} \\
\hline $\begin{array}{c}\text { (miles de pesos de } \\
1993)\end{array}$ & Primario & Secundario & Terciario & Primario & Secundario & Terciario \\
\hline $\begin{array}{l}\text { ZM Ciudad } \\
\text { de México }\end{array}$ & 885.8 & 84542.3 & 361739.6 & 27.0 & 68.2 & 82.2 \\
\hline zM Puebla-Tlaxcala & 45.2 & 10995.3 & 24297.8 & 1.4 & 8.9 & 5.5 \\
\hline zM Toluca & 611.1 & 12748.3 & 16177.1 & 18.7 & 10.3 & 3.7 \\
\hline zM Querétaro & 117.9 & 6664.0 & 13545.0 & 3.6 & 5.4 & 3.1 \\
\hline zM Cuernavaca & 661.3 & 3608.3 & 9579.7 & 20.2 & 2.9 & 2.2 \\
\hline zM Pachuca & 0.3 & 378.2 & 4812.5 & 0.0 & 0.3 & 1.1 \\
\hline zM Cuautla & 778.1 & 432.7 & 2712.7 & 23.8 & 0.3 & 0.6 \\
\hline zM Tlaxcala & 14.2 & 545.4 & 1509.3 & 0.4 & 0.4 & 0.3 \\
\hline zM Tulancingo & 9.5 & 149.9 & 1731.5 & 0.3 & 0.1 & 0.4 \\
\hline
\end{tabular}


Continúa cuadro A.1...

\begin{tabular}{|c|c|c|c|c|c|c|}
\hline \multirow{3}{*}{$\begin{array}{c}\text { Zona } \\
\text { Metropolitana } \\
\text { (miles de pesos de } \\
\text { 1993) }\end{array}$} & \multicolumn{6}{|c|}{ Producto Interno Bruto, 1998} \\
\hline & \multicolumn{3}{|c|}{ Total } & \multicolumn{3}{|c|}{$\%$} \\
\hline & Primario & Secundario & Terciario & Primario & Secundario & Terciario \\
\hline zM Tula & 2.7 & 2603.0 & 1224.6 & 0.1 & 2.1 & 0.3 \\
\hline zM Apizaco & 150.0 & 806.8 & 1922.6 & 4.6 & 0.7 & 0.4 \\
\hline $\begin{array}{l}\text { zM San Martín } \\
\text { Texmelucan }\end{array}$ & 0.0 & 561.9 & 874.1 & 0.0 & 0.5 & 0.2 \\
\hline Total metropolitano & 3276.2 & 124036.2 & 440126.2 & 100.0 & 100.0 & 100.0 \\
\hline Total región Centro & 14318.2 & 133415.5 & 461974.6 & & & \\
\hline $\begin{array}{l}\text { Porcentaje } \\
\text { metropolitano } \\
\text { de la región Centro }\end{array}$ & 22.9 & 93.0 & 95.3 & & & \\
\hline
\end{tabular}

Fuente: elaboración propia con base en (Sedesol, 2004; Conapo, 2002; INEGI, 2004).

\section{Bibliografía}

Aroca, Patricio (2004), "Migración interregional en Chile. Modelos y resultados 1987-2002", Notas de Población, núm. 78, Cepal, Santiago de Chile, pp. 97-154.

Arroyo, Mercedes (2001), "La contraurbanización: un debate metodológico y conceptual sobre la dinámica de las áreas metropolitanas", Papeles de Población, año 7, núm. 30, Universidad Autónoma del Estado de México, Toluca, pp. 93-129.

Camas, Javier (1999) (comp.), ¿Descentralización o desarticulación urbana?, Serie Antologías Universitarias, Instituto de Investigaciones José María Luis Mora-CIESAS, México.

Cepal (Comisión Económica para América Latina y el Caribe) (2007), Panorama Social de América Latina, CEPAL, Santiago de Chile.

Conapo (Consejo Nacional de Población) (2002), La situación demográfica de México 2000, Conapo, México.

Corona, Reina y José Rodolfo Luque (1992), “Cambios recientes en los patrones migratorios a la Zona Metropolitana de la Ciudad de México (zMCM)", Estudios Demográficos y Urbanos, vol. viI, núms. 2 y 3, El Colegio de México, México, pp. 575-586. 
Chávez, Ana y Sandra Savenberg (1995), "Nuevo Horizonte de la migración en el centro de México", Estudios Demográficos y Urbanos, vol. x, núm. 2, El Colegio de México, México, pp. 295-345.

Chávez, Ana y Julio Guadarrama (2000), "La transformación económica y migratoria de la región Centro de México en el contexto de la crisis", EURE Revista Latinoamericana de Estudios Urbano Regionales, vol. xxvi, núm.78, Instituto de Estudios Urbanos, Pontificia Universidad Católica de Chile, Santiago de Chile, pp. 5-36.

Chávez, Ana. y Julio Guadarrama (2004), "La región central de México en transición: tendencias económicas y migratorias a finales del milenio", en Adrián Aguilar (coord.), Procesos metropolitanos y grandes ciudades. Dinámicas recientes en México y otros paises, $\mathrm{H}$. Cámara de Diputados, LIX Legislatura, Instituto de GeografíaCRIM-PUeC-Unam-CONACYT-Miguel Ángel Porrúa, México, pp. 147-187.

De la Garza, Enrique (1994), "Reestructuración espacial y reconversión industrial”, en Mario Bassols (coord.), Campo y Ciudad en una era de transición, UAM-Iztapalapa, México, pp. 47-67.

Escamilla, Irma (2003), "Reestructuración económica y mercado laboral urbano en la región Centro", en Adrián Guillermo Aguilar (coord.), Urbanización, cambio tecnológico y costo social. El caso de la Región Centro de México, Instituto de Geografía-unam-Conacyt-Miguel Ángel Porrúa, México, pp. 227-271.

Garza, Gustavo (1990), "El carácter metropolitano de la urbanización en México, 1900-1988", Estudios Demográficos y Urbanos, vol. I 5 , núm.1, El Colegio de México, México, pp. 37-59.

Garza, Gustavo (1999), “Globalización económica, concentración metropolitana y políticas urbanas en México", Estudios Demográficos y Urbanos, vol. I4, núm. 2, El Colegio de México, México, pp. 269-311.

Geyer, Hermanus (1996), "Expanding the theoretical foundation of the concept of differential urbanization", Tijdschrift voor Economische en Sociale Geografie, vol. 87, núm 1, Wiley-Blackwell, Utrecht, pp. 44-59. 
Geyer Hermanus y Thomas Kontuly, (1993), "A theoretical foundation for the concept of differential urbanization", International Regional Science Review, vol. 15, núm. 2, Sage Publications, Arizona, pp. 157-177.

Graizbord, Boris y Alejandro Mina (1994), “Los ámbitos geográficos del componente migratorio de la ciudad de México", Estudios Demográficos y Urbanos, vol. 9, núm. 3, El Colegio de México, México, pp. 609-628.

Graizbord, Boris (1984), "Perspectivas de una descentralización del crecimiento urbano en el sistema de ciudades de México", Revista Interamericana de Planificación, vol. xviII, núm. 71, Sociedad Interamericana de Planificación, Guatemala, pp. 36-58.

Granados, José Aurelio (2007), "Las corrientes migratorias en las ciudades contiguas a la Zona Metropolitana de la Ciudad de México: El caso de la aglomeración urbana de Pachuca”, Estudios Demográficos y Urbanos, vol. 22, núm. 3, El Colegio de México, México, pp. 619-649.

Guzmán, José, Jorge Rodríguez, Jorge Martínez, Juan Manuel Contreras y Daniela Guzmán (2006), "La demographie de l'Amerique Latine et de la Caraïbe depuis 1950", Population- $F$, vol. 61, núm. 5-6, Institut Nacional d'Etudes Demographiques, Paris, pp. 623-734.

Henderson, Vernon (2000), "The effects of urban concentration on economic growth", documento de trabajo 7503, National Bureau of Economic Research, Cambridge, Massachusetts.

INEGI (Instituto Nacional de Estadística, Geografía e Informática) (1999), Censos Económicos 1999, inegr, Aguascalientes, México.

INEGI (Instituto Nacional de Estadística, Geografía e Informática) (2000), Sistema de Integración Territorial, ITER, INEGI, Aguascalientes, México.

INEGI (Instituto Nacional de Estadística, Geografía e Informática) (2001), XII Censo General de Población y Vivienda 2000, INEgI, Aguascalientes, México. 
INEGI (Instituto Nacional de Estadística, Geografía e Informática) (2004), Censos Económicos 2004, Inegi, Aguascalientes, México.

INEGI (Instituto Nacional de Estadística, Geografía e Informática) (2005), Sistema de Integración Territorial, ITER, INEGI, Aguascalientes, México.

INEGI (Instituto Nacional de Estadística, Geografía e Informática) (2006), II Conteo de Población y Vivienda 2005, InEgI, Aguascalientes, México.

Morelos, José (2005), “Tendencias y cambio estructural de la participación de la mujer en la actividad económica en 1970 y 2000", Estudios Demográficos y Urbanos, vol. 20, núm. 1, El Colegio de México, México, pp. 125-150.

Negrete, María Eugenia (1990), "La migración hacia la ciudad de México: un proceso multifacético", Estudios Demográficos y Urbanos, vol. 5, núm. 3, El Colegio de México, México, pp. 641-654.

Negrete, María Eugenia (1999), "Desconcentración poblacional en la región Centro de México”, Estudios Demográficos y Urbanos, vol. 14, núm. 2, El Colegio de México, México, pp. 313-352.

Olivera, Guillermo y Julio Guadarrama (2002), "El ciclo de reestructuración industrial y territorial de la región Centro de México", en Javier Delgadillo y Alfonso Iracheta (coords.), Actualidad de la investigación regional en el México Central, CRIM-UNAM-El Colegio Mexiquense-El Colegio de Tlaxcala-Plaza y Valdés, México, pp. 141-194.

Pinto da Cunha, José Marcos (2002), Urbanización, territorio y cambios socioeconómicos estructurales en América Latina y el Caribe, Serie Población y Desarrollo, núm. 30, CEPAL, Chile.

Polèse, Mario (1998), Economía urbana y regional. Introducción a la relación entre territorio y desarrollo, Libro universitario regional, Cartago.

Rodríguez, Jorge (2008), "Distribución espacial, migración interna y desarrollo en América Latina y El Caribe", Revista de la CEPAL 96, Santiago de Chile, pp. 135-155. 
Rodríguez, Jorge (2004), Migración interna en América Latina y El Caribe: estudio regional del periodo 1980-2000, serie Población y Desarrollo, núm. 50, Cepal, Santiago de Chile.

Rodríguez, Jorge (2002) Distribución territorial de la población de América Latina y el Caribe: tendencias, interpretaciones y desafios para las políticas públicas, Serie Población y Desarrollo núm. 32, CEPAL, Santiago de Chile.

Ruiz, Crescencio (1999), "Población y migraciones rurales en México: Hipótesis para otro siglo", Economía, Sociedad y Territorio, vol. II, núm. 5, El Colegio Mexiquense, Zinacantepec, pp. 239-257.

Sedesol, Conapo, inegi (2004), Delimitación de zonas metropolitanas, Secretaría de Desarrollo Social, Consejo Nacional de Población, Instituto Nacional de Estadística Geografía e Informática, México.

Unfpa (Fondo de Población de las Naciones Unidas) (2007), Estado de la Población Mundial 2007, Fondo de Población de las Naciones Unidas, Nueva York.

Recibido: 4 de mayo de 2010. Reenviado: 8 de agosto de 2011. Aceptado: 18 de octubre de 2011.

Guillermo Olivera-Lozano. Es investigador y secretario académico del Centro Regional de Investigaciones Multidisciplinarias (CRIM) de la Universidad Nacional Autónoma de México (UNAM). Realizó estudios de licenciatura y maestría en geografía y actualmente cursa el doctorado en urbanismo, todos ellos en la UNAM. Sus líneas de investigación son reestructuración económica y su impacto territorial, así como la planeación y el análisis del desarrollo urbano. Ha publicado varios artículos en revistas y capítulos en libros sobre los temas señalados. Entre sus publicaciones más recientes se encuentran: "Deterioro económico y emigración en México, 1990-2004", en Comercio Exterior, Vol. 58, núm. 7, Bancomext, México, pp. 543-558 (2008); "Especialización en comercio y servicios al consumidor en Morelos, 1980-2003”, en Gustavo Garza y Jaime Sobrino (eds.), Evolución del sector servicios en las ciudades y regiones de México, El Colegio de México, México, pp. 287-384 (2009). 
Carlos Galindo-Pérez. Es licenciado y maestro en Geografía por la Universidad Nacional Autónoma de México (UNAM), cursa actualmente el doctorado en urbanismo en esa misma institución. Actualmente es investigador en el Centro Regional de Investigaciones Multidisciplinarias (CRIM) en esa misma Universidad, como especialista en el análisis territorial de procesos urbanos y poblacionales con base en el uso de Sistemas de Información Geográfica. Ha sido profesor de asignatura en el Instituto Mora y en el programa de posgrado de la Facultad de Economía de la UNAM. Entre sus publicaciones más recientes sobresale el artículo: "Los espacios emergentes de la dinámica rural-urbana”, Problemas del Desarrollo, vol. 37, núm. 147, Instituto de Investigaciones Económicas de la UNAM, México, pp. 187-216 (2006). 